\title{
Cohesive Traction-Separation Laws for Tearing of Ductile Metal Plates
}

\section{Citation}

Nielsen, K. L., and John W. Hutchinson. 2012. Cohesive traction-separation laws for tearing of ductile metal plates. International Journal of Impact Engineering 48:15-23.

\section{Published Version}

doi:10.1016/j.jimpeng.2011.02.009

\section{Permanent link}

http://nrs.harvard.edu/urn-3:HUL.InstRepos:10163031

\section{Terms of Use}

This article was downloaded from Harvard University's DASH repository, and is made available under the terms and conditions applicable to Open Access Policy Articles, as set forth at http:// nrs.harvard.edu/urn-3:HUL.InstRepos:dash.current.terms-of-use\#OAP

\section{Share Your Story}

The Harvard community has made this article openly available.

Please share how this access benefits you. Submit a story.

\section{Accessibility}




\title{
Cohesive Traction-Separation Laws for Tearing of Ductile Metal Plates
}

\author{
K.L. Nielsen ${ }^{1,2}$ and J.W. Hutchinson ${ }^{2}{ }^{*}$ \\ ${ }^{1}$ Department of Mechanical Engineering, Solid Mechanics, Technical University of Denmark, \\ DK-2800 Kgs. Lyngby, Denmark \\ ${ }^{2}$ School of Engineering and Applied Sciences, Harvard University, Cambridge, MA, United States
}

\begin{abstract}
The failure process ahead of a mode I crack advancing in a ductile thin metal plate or sheet produces plastic dissipation through a sequence of deformation steps that include necking well ahead of the crack tip and shear localization followed by a slant fracture in the necked region somewhat closer to the tip. The objective of this paper is to analyze this sequential process to characterize the traction-separation behavior and the associated effective cohesive fracture energy of the entire failure process. The emphasis is on what is often described as plane stress behavior taking place after the crack tip has advanced a distance of one or two plate thicknesses. Tractionseparation laws are an essential component of finite element methods currently under development for analyzing fracture of large scale plate or shell structures. The present study resolves the sequence of failure details using the Gurson constitutive law based on the micromechanics of the ductile fracture process, including a recent extension that accounts for damage growth in shear. The fracture process in front of an advancing crack, subject to overall mode I loading, is approximated by a 2D plane strain finite element model, which allows for an intensive study of the parameters influencing local necking, shear localization and the final slant failure. The deformation history relevant to a cohesive zone for a large scale model is identified and the traction-separation relation is determined, including the dissipated energy. For ductile structural materials, the dissipation generated during necking prior to the onset of shear localization is the dominant contribution; it scales with the plate thickness and is mesh-independent in the present numerical model. The energy associated with the shear localization and fracture is secondary; it scales with the width of the shear band, and inherits the finite element mesh dependency of the Gurson model. The cohesive tractionseparation laws have been characterized for various material conditions.
\end{abstract}

Keywords: Cohesive zone model, Gurson model, Shear localization, Slant fracture, Ductile plates.

This paper is dedicated to Professor S. R. Reid on the occasion of his $65^{\text {th }}$ birthday in celebration of his contributions to the deformation and failure of ductile metal components and structures.

\footnotetext{
* Corresponding author:
} 
Tel: (01) 617-495-2848

Email address: hutchinson@husm.harvard.edu (J.W. Hutchinson) 


\section{Introduction}

It is widely recognized that finite element analysis to determine extensive crack growth in large plate or shell structures cannot be expected to resolve details of the fracture process. For tough ductile structural alloys, meshes that are fine compared to the thickness of the plate or shell would be required to capture necking behavior prior to the onset of appreciable material damage. An accurate resolution of the fracture process itself for ductile materials that fail by the mechanism of void nucleation, growth and coalescence typically would require the mesh to scale with the dominant void spacing (e.g. 100 microns). Mesh resolution on this scale is possible for test specimens and small components but not for larger structures. The in-plane element size used in the analysis of large plate or shell structures is usually at least several plate thicknesses and therefore far larger than the size required to even resolve local necking. One approach to bridging the multiple scales is to incorporate a Cohesive Zone Model in the large scale finite element formulation which in the present context would characterize the failure process beyond the onset of necking ahead of the advancing crack tip. The utility of the cohesive zone for the analysis of large plate and shell structures has been amply demonstrated and codes based on newer X-FEM approaches that embed a cohesive zone are becoming available.

The incorporation of a cohesive zone in a large scale computation requires the tractionseparation law to provide a reasonable approximation to the failure process zone associated with crack advance. In principle, a cohesive zone model could be calibrated against experimental crack propagation data or it could be theoretically modeled using a numerical method that resolves the fracture process. In practice, it is likely that some combination of experimental and theoretical methods will be required to establish effective characterizations. This paper is an attempt to characterize the cohesive zone for the analysis of extensive mode I crack advance in plates comprised of tough ductile structural alloys. The work here builds on earlier studies addressing tearing in thin metal sheets and plates [1,2], including work which specifically addresses the role of necking localization in contributing to plastic dissipation in the effective cohesive zone [3] and work which applies a cohesive zone for the analysis of extensive tearing under plane stress conditions [4].

After the crack tip has advanced by one or two plate thicknesses, the failure process ahead of a mode I crack propagating in a ductile thin metal plate or sheet produces plastic dissipation through a sequence of deformation steps that includes local necking well ahead of the tip, a smaller scale localization in the neck somewhat closer to the tip in the form of a shear band or a "bath tub" band leading to final separation just ahead of the tip [3]. The sequence considered in this paper is 
depicted in Fig. 1 and gives rise to the commonly observed slant fracture. The final slant fracture of a tearing test is seen in Fig. 2. This picture was taken from the paper by Simonsen and Törnqvist [5] who carried out a set of large scale tests for mode I crack advance in ductile aluminum and steel plates with cracks propagating up to 30-40 times the plate thickness. The objective of this paper is to analyze the sequential process governing this failure mode and thereby characterize the tractionseparation behavior and associated cohesive fracture energy of the entire failure process. The phenomenon seen in Fig. 2 in which the fracture slant "flips" back and forth from one roughly 45 degree orientation to the other, after growth on the order of 10 times the plate thickness, has not been resolved in the present study. However, the numerical results will show a second "inactive" shear-band co-existing with the band governing the crack advance which may be relevant.

The initiation of crack advance from a blunted tip is not addressed in this paper. Rather, it is imagined that the crack tip has already advanced by several plate thicknesses such that the steadystate deformation/fracture sequence ahead of the tip depicted in Fig. 1 is fully established. The present study resolves the sequence of failure details using a finite strain version of the Gurson [6] constitutive law for the ductile damage process, including a recent extension accounting for damage growth in shear [7]. The fracture process in front of an advancing crack, subject to mode I loading, is approximated by a $2 \mathrm{D}$ plane strain finite element model. The portion of the deformation history relevant to the cohesive zone for a large scale model is identified and the traction-separation relation and the dissipated energy are determined. In addition, two distinct contributions to the dissipated energy will be indentified and computed: the first due to necking, and the second due to shear localization and fracture. For ductile structural plate materials, the dissipation generated during necking prior to the onset of shear localization will be found to be the dominant contribution. It is significant that this contribution will be seen to be mesh-independent in the numerical model and to scale precisely with the plate thickness. The smaller dissipation contribution associated with shear localization and shear fracture scales with the element size, and this mesh sensitivity will be addressed.

The paper is structured as follows. The material model and the plane strain finite element model are outlined in Section 2. Results are presented in Section 3, including a comparison of the onset of shear localization from the finite element analysis and that from an analytical shear band analysis. Conclusions are given in Section 4 along with discussion of information that will be required to implement cohesive zone modeling in addition to the present results.

\section{Material and finite element models}


Finite strain, plane strain finite element simulations have been reported in the literature for many years. The present work builds upon simulations of necking, shear band localization and fracture of ductile metals under tensile loading, as addressed, for example, in [8-13], with specific application to the characterization of cohesive zones for plates and shells as described in the Introduction. Where clarity of the paper is not sacrificed, previously published details of the constitutive model and the finite strain elastic-plastic formulation will be omitted and cited.

\subsection{Material model}

The central features of the model presented by Gurson [6] and extended in [7] to account for damage growth in shear are as follows. The model is an isotropic formulation that employs the three invariants of the Cartesian components of the Cauchy (true) stress, $\sigma_{i j}$ : the mean stress, $\sigma_{m}=\sigma_{k k} / 3$, the effective stress, $\sigma_{e} \equiv \sqrt{3 J_{2}}=\sqrt{3 s_{i j} s_{i j} / 2}$, where $s_{i j}=\sigma_{i j}-1 / 3 \sigma_{k k} \delta_{i j}$ is the stress deviator, and a third invariant defined below in Eq. (4). The yield surface is specified by

$$
F\left(\sigma_{e}, \sigma_{m}, f\right)=\left(\frac{\sigma_{e}}{\sigma_{M}}\right)^{2}+2 q_{1} f \cosh \left(\frac{3 q_{2}}{2} \frac{\sigma_{m}}{\sigma_{M}}\right)-\left(1+\left(q_{1} f\right)^{2}\right)
$$

where the current state is characterized by $f$, the damage parameter which can be interpreted as an effective void volume fraction, and $\sigma_{M}$ is the current effective true stress governing flow of the damage-free base material which is specified below. The fitting parameters, $q_{1}$ and $q_{2}$, were introduced in $[8,14]$. All quantities not labeled with the subscript $M$ represent overall quantities associated with the damaged material. Normality implies that the plastic strain rate, $D_{i j}^{P}$, is given by

$$
D_{i j}^{P}=\frac{1}{h} P_{i j} P_{k l} \dot{\sigma}_{k l}
$$

where

$$
P_{i j}=\frac{\partial F}{\partial \sigma_{i j}}=\frac{3 s_{i j}}{\sigma_{M}{ }^{2}}+\frac{f q_{1} q_{2}}{\sigma_{M}} \sinh \left(\frac{3 q_{2} \sigma_{m}}{2 \sigma_{M}}\right) \delta_{i j}
$$

In finite strain formulations, $\dot{\sigma}_{i j}$ is identified with the Cartesian components of the Jaumann rate of stress. The expression for the hardening modulus, $h$, is given in the references cited above.

The original Gurson model predicts no damage growth and monotonic hardening in pure shear. The extension outlined below was proposed in [7] to account for damage growth and softening in shear. In addition to $\sigma_{m}$ and $\sigma_{e}$, the extended model employs the third stress invariant 


$$
J_{3}=\operatorname{det}(\mathbf{s})=\frac{1}{3} S_{i j} s_{i k} S_{j k}=\left(\sigma_{I}-\sigma_{m}\right)\left(\sigma_{I I}-\sigma_{m}\right)\left(\sigma_{I I I}-\sigma_{m}\right)
$$

where the expression on the right is couched in terms of principal stresses, assumed to be ordered as $\sigma_{I} \geq \sigma_{I I} \geq \sigma_{I I I}$. The non-dimensional invariant,

$$
\omega(\boldsymbol{\sigma})=1-\left(\frac{27 J_{3}}{2 \sigma_{e}^{3}}\right)^{2},
$$

lies in the range, $0 \leq \omega \leq 1$, with $\omega=0$ for all axi-symmetric stress states,

$$
\sigma_{I} \geq \sigma_{I I}=\sigma_{I I I} \text { or } \sigma_{I}=\sigma_{I I} \geq \sigma_{I I I},
$$

and $\omega=1$ for all states comprised of a pure shear stress plus a hydrostatic contribution,

$$
\sigma_{I}=\tau+\sigma_{m}, \sigma_{I I}=\sigma_{m}, \sigma_{I I I}=-\tau+\sigma_{m} \quad(\tau>0)
$$

The original Gurson model was formulated and calibrated based on the mechanics of void growth under axi-symmetric stress states. The extension does not alter the model for these states, nor does it alter the yield condition (1). The extension modifies the predicted growth of the damage parameter, $f$, for states with non-zero $\omega(\boldsymbol{\sigma})$. In particular, a contribution to damage growth under pure shear stress states is accounted for in the extension whereas the original Gurson model predicts no change in damage for states having $\sigma_{m}=0$. The extension of the Gurson model posits

$$
\dot{f}=(1-f) D_{k k}^{p}+k_{\omega} f \omega(\boldsymbol{\sigma}) \frac{S_{i j} D_{i j}^{p}}{\sigma_{e}}
$$

The first contribution is that incorporated in the original model while the second is the crux of the extension. In a state of pure shear, (8) gives $\dot{f}=k_{\omega} f \dot{\gamma}^{P} / \sqrt{3}$, where $\dot{\gamma}^{P}$ is the plastic shear strain rate and $k_{\omega}$ is the shear damage coefficient, the sole new parameter in the extended model. In the extension, $f$ is no longer directly tied to the plastic volume change. Instead, it must be regarded either as an effective void volume fraction or simply as a damage parameter, as it has been, for example, when the Gurson model is applied to materials with distinctly non-spherical voids. Further discussion and illustrations of the extension are given in $[7,14,15]$. Included is the specification of the widely used technique that accelerates damage from $f=f_{c}$ to $f=f_{f}$, at which point the material element is eliminated [12]. The equations above fully specify the constitutive model of the material; the remaining equations specifying for example the incremental moduli are listed in [7] using the same notation as in this paper. ${ }^{1}$ The primary damage parameters are the initial

\footnotetext{
${ }^{1}$ The sign of the second term on the right hand side of Eq. (13) in [7] should be minus not plus.
} 
void volume fraction, $f_{0}$, and the shear damage coefficient, $k_{\omega}$; these will be varied in the simulations presented in the sections on results.

The uniaxial true-stress versus logarithmic strain curve for the undamaged material is taken as

$$
\varepsilon= \begin{cases}\frac{\sigma_{M}}{E}, & \sigma_{M}<\sigma_{y} \\ \frac{\sigma_{y}}{E}\left(\frac{\sigma_{M}}{\sigma_{y}}\right)^{1 / N}, & \sigma_{M} \geq \sigma_{y}\end{cases}
$$

with $\sigma_{y}$ as the initial yield stress. The material parameters used in the simulations are given in Table I.

\subsection{Finite strain formulation}

A Lagrangian framework is used for the finite strain formulation with the undeformed body as reference and coordinates in the deformed state denoted by $x_{i}$, as detailed, for example, by $[17,18]$. Using a convected coordinate formulation of the governing equations, the components of vectors and tensors are obtained by dot products with the appropriate base vectors. The constitutive relation provides the incremental relation between the contravariant components of the Kirchhoff stress rate, $\dot{\tau}^{i j}$, and the covariant components of the Lagrangian strain rate, $\dot{\eta}_{i j}$, as

$$
\dot{\tau}^{i j}=L^{i j k l} \dot{\eta}_{k l}
$$

with plastic loading and elastic unloading branches for the incremental moduli, $L^{i j k l}$. The principle of virtual work for the incremental problem is

$$
\int_{V}\left(\dot{\tau}^{i j} \delta \eta_{i j}+\tau^{i j} \dot{u}_{, j}^{k} \delta u_{k, i}\right) \mathrm{d} V=\int_{S} \dot{T}^{i} \delta u_{i} \mathrm{~d} S-\left[\int_{S}\left(\tau^{i j} \delta \eta_{i j}-T^{i} \delta u_{i}\right) \mathrm{d} V\right]
$$

Here, $u^{i}$ and $u_{i}$ are the contravariant and covariant components of the displacement vector, $T^{i}$ is the surface traction vector per original area and the comma denotes covariant differentiation. The term in the square brackets in (11) is included as a means to eliminate residual equilibrium errors in the finite element formulation.

\subsection{Problem formulation}

After the onset of necking ahead of an advancing crack tip, the material above and below the neck will unload elastically enforcing plane strain conditions, i.e. enforcing essentially zero additional straining in the direction parallel to the crack $\left(\dot{\eta}_{33} \cong 0\right)$. Thus, the sequence of 
deformation states depicted in Fig. 1 can be well approximated by considering the 2D plane strain problem set up in Fig. 3. Two sets of boundary conditions are considered in this study as shown in Fig. 3: (i) an unconstrained condition in Fig. 3a with zero horizontal tractions on the top $\left(x_{2}=L_{0} / 2\right)$ and bottom $\left(x_{2}=-L_{0} / 2\right)$ edges, where only the middle node at $x_{1}=x_{2}=0$ is constraint to prevent free body motion, and (ii) a constrained condition in Fig. $3 \mathrm{~b}$ for which horizontal displacements of above and below the failure process zone are constrained. These conditions are modeled by imposing zero horizontal displacement along the centerline of the upper and lower part of the section. This is intended to model the maximum possible constraint on out-ofplane deflection imposed by the larger structure. For both sets of boundary conditions, the section is loaded by applying uniform increments of the vertical displacement, $\Delta / 2$, along the top and, $-\Delta / 2$, along bottom edges, while the resultant vertical force per unit depth, $F$, which is work conjugate to $\Delta$ is computed.

The geometry and loadings depicted in Fig. 3 are symmetric with respect to both the $x_{1}$-axis and the $x_{2}$-axis. If the material properties strictly shared these same symmetries, localization would first occur as a symmetric Considère neck. As the neck develops, a second localization into two equally active symmetric shear bands occurs inside the neck region [10]. The symmetric situation almost immediately gives way to asymmetric localization into one of the two bands promoted by exceedingly small perturbations or imperfections. In the present study, to promote failure in a single shear band, a very small asymmetric imperfection in the yield stress distribution has been introduced within a narrow band of width $b$ oriented at $45^{\circ}$ to the centerline. With reference to Fig. 3, the distribution in the band is chosen as

$$
\sigma_{y}^{b}\left(x_{1}, x_{2}\right)=\sigma_{y}\left[1-\beta \frac{1}{2}\left(1+\cos \left(\pi \frac{x_{2}-x_{1}}{2 b}\right)\right)\right] \quad \text { for } x_{1}-b \leq x_{2} \leq x_{2}+b
$$

Here, $\sigma_{y}$ is the initial yield stress of the material everywhere outside the band, $\beta=0.001$ is the amplitude of the imperfection, and $b=W_{0} / 10$ is the width of the imperfection. In the band, $\sigma_{y}^{b}$ is substituted for $\sigma_{y}$ in (9). The imperfection is solely introduced to promote the localization into a single shear band. Its amplitude is sufficiently small such that it does not otherwise effect the inclination of the shear band or the computed traction-separation behavior.

The notation and a representative mesh are shown in Fig. 3 for the full section. The height and width of the un-deformed section are $L_{0}$ and $W_{0}$, respectively, with $L_{0} / W_{0}=3$ for all results presented in this study. A uniform mesh of square elements of size $L^{(e)} \times L^{(e)}$ is used within the 
region that undergoes necking, shear localization and slant fracture. The effect of element size will be investigated and the element size will be reported as a fraction of the sheet thickness, $W_{0}$. Isoparametric 8 node plane elements are employed, using reduced Gaussian quadrature $(2 \times 2$ Gauss points) for the integration.

The boundary value problem posed above, including the initial distribution of yield stress, and its solution possess $180^{\circ}$ rotational symmetry about the $x_{3}$-axis such that only the region above the $x_{1}$-axis needs to be meshed. Consistent with the rotational symmetry, the boundary conditions along $x_{2}=0$ for the upper part of the finite element mesh in Fig. $3 \mathrm{c}$ are: $u_{1}\left(x_{1}, 0\right)=-u_{1}\left(-x_{1}, 0\right)$ and $u_{2}\left(x_{1}, 0\right)=-u_{2}\left(-x_{1}, 0\right)$. These boundary conditions are applicable to strictly symmetric and antisymmetric deformations, as well as the present mixed problem. ${ }^{2}$ These conditions are imposed in the finite element code using a standard penalty approach [20].

\section{Results: Necking, shear localization and failure}

\subsection{Identification of traction-separation relation for cohesive zone}

To set the stage for the presentation of results characterizing the cohesive zone, a representative computed result in the form of the dimensionless load/depth, $F /\left(\sigma_{y} W_{0}\right)$, as a function of the normalized overall elongation, $\Delta / L_{0}$, is given in Fig. 4a. As is well known, necking begins at the maximum load. In plane strain tension the onset of necking occurs at the Considère condition, which for the present material is when the logarithmic strain attains, $\varepsilon_{L O G}=N$. Since $N=0.1$ in this example, $\varepsilon_{L O G}$, and the overall engineering strain, $\Delta / L_{0}$, differ only slightly prior to necking, and thus the onset of necking is at $\Delta_{C} / L_{0} \cong 0.1$. Beyond the Considère point, continuing deformation is localized to the neck which initially extends roughly one width, $W_{0}$, above and below the horizontal centerline of the section. As the neck develops under increasing $\Delta / L_{0}$, the load falls gradually until the onset of shear localization noted in Fig. 4a, whereupon the load begins to fall abruptly with relatively little further overall elongation. In this last stage, continuing deformation is now localized to the shear band with almost no additional deformation in the neck outside the band. The problem studied in this paper is thus characterized by two fundamental localization phenomena, necking and shear banding, the latter contained within the former. As

\footnotetext{
${ }^{2}$ Analogous boundary conditions were exploited by Tvergaard [19] in his study of necking and shear localization in three dimensional bars pulled in tension.
} 
shearing progresses in the band, damage increases until shear failure occurs with complete separation.

The following issue is now addressed: What part of the load-elongation behavior in Fig. 4a is relevant to the characterization of the cohesive zone? The cohesive zone in a large scale finite element model should represent that part of the behavior that the elements cannot capture. Plate or shell elements ahead of a long crack can capture behavior up to the onset of necking and they can correctly represent elastic unloading once necking begins. But, they cannot capture the sequence of deformations in the neck beyond the onset of the necking localization. It is this part of the loadelongation behavior in Fig. 4a which must be employed to characterize the cohesive zone. The traction-separation curve for the cohesive zone extracted from Fig. $4 \mathrm{a}$ is plotted in Fig. $4 \mathrm{~b}$ as $F /\left(\sigma_{y} W_{0}\right)$ (normalized traction per original area) as a function of the additional normalized separation, $\delta / W_{0}$, with $\delta=\Delta-\Delta_{C}$. The nominal Considère stress is $\sigma_{C}=F_{M A X} / W_{0}$. Note that the relation between $F /\left(\sigma_{y} W_{0}\right)$ and $\delta / W_{0}$ will be independent of the height, $L_{0}$, of the section if one ignores the elastic unloading contraction of the sections above and below the neck after the onset of necking. The elastic unloading contraction can be subtracted off but it is so small that its influence is negligible.

This example, which is typical for tough ductile alloys, already makes it clear that the cohesive work of separation, $\int T \mathrm{~d} \delta \equiv \Gamma_{0}=\Gamma_{I}+\Gamma_{I I}$ (with $T$ as the nominal traction) is primarily due to the energy dissipated between the onset of necking and the onset of shear localization, $\Gamma_{I}$. The dissipation occurring subsequent to the onset of shear localization, $\Gamma_{I I}$, is relatively small. These findings will be elaborated on below.

\subsection{The onset of shear localization}

Rice [21] established the analytical condition for the onset of shear localization as a bifurcation condition depending on the local state of deformation and stress. The curves in Figs. 5a,c are predictions for effective plastic strain at the onset of shear band bifurcation from the state of plane strain tension as a function of $f_{0}, k_{\omega}$ and $N$. The details of the calculations underlying these results have been given in [7] and will not be repeated here. The band orientation is within one or two degrees from $45^{\circ}$ to the tensile axis. These results are compared to the finite element model in Figs. 5b,d by mapping the circular data points in Figs. 5a,c from the shear band bifurcation analysis onto the predicted effective plastic strain evolution in an element at the center of the neck where 
shear localization begins. As seen in Figs. 5b,d, the element undergoes an abrupt increase in plastic strain at the onset of shear localization. The agreement between the analytical bifurcation condition for a shear band and the onset of the localization band in the finite element calculation is remarkably good even though the state of deformation at the center of the neck in the finite element model prior to shear localization is not precisely plane strain tension. Some additional stress triaxiality develops during necking in the finite element model which accelerates the onset of shear localization. For the cases in Figs. 5c,d, the additional triaxiality accounts for the small discrepancy between the bifurcation prediction and the finite element results.

After the shear band forms in the center of the neck it spreads towards edges in a direction at roughly $45^{\circ}$ to the centerline. Once the band reaches the edges, plastic straining becomes almost entirely localized to the band such that the overall elongation is abruptly curtailed (c.f. Fig. 4). In the present finite element model, the thickness of the band is set by the size of the elements within the neck. The band is essentially one element thick, as elements on either side of the band undergo elastic unloading. The element size-dependence of the traction-separation behavior will be explored in Section 3.4.

\subsection{Two boundary conditions and initial imperfections}

The two limiting boundary constraints introduced in Section 2.3 and depicted in Fig. 3 have been considered to provide insight into conditions that will be encountered in applying a cohesive zone model for the fracture analysis of large plate structures. The constrained case represents the limit where the sections of the plate and the supporting structure above and below the cohesive zone do not permit any overall out-of-plane displacement across the zone, while the unconstrained case is the limit where there is no resistance to an overall out-of-plane displacement across the zone. In the present model, these limiting conditions will depend on $L_{0}$, but there is very little difference in the overall traction-displacement behavior for the two limits when $L_{0} / W_{0}=3$, as seen in Fig. 6. Prior to the onset of shear localization, the responses for the two cases are indistinguishable. Following shear localization, more energy is dissipated in the constrained case, but the difference is very small. This outcome is fortunate for applications of cohesive models to ductile plates because it implies that the zone characteristics can be specified without regard for the out-of-plane constraint. All the results which follow have been computed with the unconstrained boundary conditions.

\subsection{Traction-separation and cohesive energy}


The maximum nominal traction, $T_{M A X}=F_{M A X} / W_{0}$, can be identified as the Considère load per original area at the onset of necking, where the cohesive separation process begins, $\delta=0$. As can be seen in Figs. 4 and 6, the traction falls gradually until the onset of shear localization where it begins a precipitous fall. In most applications of cohesive traction-separation laws, the two most important features are the maximum traction and the work/original area of the tractions, $\Gamma_{0}=\Gamma_{I}+\Gamma_{I I}=\int T \mathrm{~d} \delta$. But, as discussed in [22], the exact details of $T$ versus $\delta$ will generally not be essential as long $T_{M A X}$ and $\Gamma_{0}$ are accurately reproduced and the functional form is faithful to the general features seen in Figs. 4 and 6. In the remainder of this paper, the results presented will highlight the dimensionless cohesive dissipation energy, $\Gamma_{0} /\left(\sigma_{y} W_{0}\right)$, while $T_{M A X}$ can be computed by elementary methods. For the power-law hardening material in (9) with no damage, the Considère condition for plane strain tension gives

$$
\frac{T_{M A X}}{\sigma_{y}}=\frac{2}{\sqrt{3}}\left(\frac{2}{\sqrt{3}} \frac{N E}{\sigma_{y}}\right)^{N} e^{-N}
$$

if elastic compressibility is ignored. Damage reduces the maximum traction but only slightly as will be seen in the results presented below.

The role of the finite element mesh on the results of interest are brought out by Fig. 7a where the traction-separation behavior is presented for one specific material case $\left(N=0.1, f_{0}=0.01\right.$ and $\left.k_{\omega}=0\right)$ and for four meshes with square elements of dimensions, $L^{(e)} \times L^{(e)}$, using the normalization, $L^{(e)} / W_{0}$. Prior to shear localization, there is essentially no mesh dependence because all four meshes are fine enough to accurately predict the necking response. Similarly, assuming the stresses and strains in the neck to be adequately resolved, the onset of shear localization at the center of the neck is not very sensitive to meshing, because the onset condition depends on local stresses and strains and not on their gradients. However, the subsequent growth of the shear localization and shear failure is directly tied to element size, as discussed earlier. No length scale has been introduced in the material model that would limit strain gradients. The shear band has essentially one element across its width and thus has an approximate thickness, $L^{(e)}$. Figs. 7b-e clearly indicates that the final stage of the traction-separation process depends strongly on the element size - the larger the element, the thicker the localization band and the more energy is dissipated. The mesh in Fig. $7 \mathrm{~b}$ with the largest elements is too crude to even qualitatively capture the formation of a realistic shear band. 
A systematic study of the dependence of the cohesive work of separation on $L^{(e)} / W_{0}$ is presented in Fig. 8. The limit as $L^{(e)} / W_{0} \rightarrow 0$ in Fig. 8 is obtained by extrapolation of the computed points as indicated. This limit is the prediction for $\Gamma_{I} /\left(\sigma_{y} W_{0}\right)$ obtained as the area under the traction-separation curve computed between the onset of necking and the onset of shear localization at the center of the neck. Included in Fig. 8 as square points on the ordinate are the predictions from an estimate of $\Gamma_{I}$ computed in uniform plane strain tension for the energy absorbed between the Considère load and the onset of shear localization from the shear band analysis. Mainly due to the triaxiality increase above plane strain tension, the extrapolated results for $\Gamma_{I}$ from the finite element calculations fall below the estimates, but the difference is quite small. As noted earlier, $\Gamma_{I}$ is insensitive to the finite element mesh as long as it resolves the neck. The cohesive energy can be partitioned precisely into the contribution, $\Gamma_{I}$, between the onset of necking (the intercept on the ordinate) and the onset of shear localization, and the mesh-dependent contribution, $\Gamma_{I I}$, from the final stage following the onset of shear localization. From Fig. 8 it can be seen that $\Gamma_{I I} /\left(\sigma_{y} W_{0}\right) \cong \alpha L^{(e)} / W_{0}$ where $\alpha$ lies between 1 and 2 . This implies $\Gamma_{I I} \cong \alpha \sigma_{y} L^{(e)}$, consistent with the expectation that the energy/area dissipated in the final stage is on the order of $\sigma_{y} \times$ a strain of order unity $\times$ the thickness of the shear band. It is also evident that the dominant contribution to the cohesive energy is from the onset of necking to the onset of shear localization. Moreover, this dominant contribution scales precisely with the thickness of the plate.

The three most important material parameters influencing the cohesive energy are $N, f_{0}$ and $k_{\omega}$. Trends showing the dependence on $N$ and $k_{\omega}$ are presented in Fig. 9 for a mesh with $L^{(e)}=W_{0} / 96$. For materials with the tensile stress-strain behavior (9), the only other dimensionless parameters affecting the traction-separation behavior are $\sigma_{y} / E, f_{C}$ and $f_{f}$. The influence of $\sigma_{y} / E$ is displayed in Figs. 10a,b. The dependence is directly related to the dependence of the maximum load on $\sigma_{y} / E$ as reflected by (13). This dependence can be captured quite accurately if one uses the nominal stress (13) at the Considère condition, $\sigma_{C} \equiv T_{M A X}$, in place of $\sigma_{y}$ in the normalization of the traction, i.e. $F /\left(\sigma_{C} A_{0}\right)$. This assertion is demonstrated in Fig. 10c where the curves for $\Gamma_{0} /\left(\sigma_{C} A_{0}\right)$, with the strain hardening $N \in[0.05,0.2]$, show little dependence on $\sigma_{y} / E$. This could also be seen from the traction-separation curves as they nearly collapse to a single curve when this alternative normalization was used, for a given strain hardening, $N$. A few additional 
calculations in which the coalescence parameters $f_{C}$ and $f_{f}$ are varied over the ranges, $0.1 \leq f_{C} \leq 0.2$ and $0.35 \leq f_{f} \leq 0.45$, have been carried out to assess their influence. The maximum variation of $\Gamma_{I I}$ is approximately $20 \%$. Because $\Gamma_{I I}$ is such a small fraction of the total work of separation, $\Gamma_{0}$, one concludes that the primary results of interest in this study depend very weakly on the coalescence parameters.

\section{Conclusions and extensions}

The energy/area, $\Gamma_{0}$, associated with a cohesive zone model of ductile plates subject to mode I tearing has been identified as the energy dissipated during necking, shear localization and slant fracture following the onset of necking in the zone ahead of the crack tip. The present work provides a detailed treatment of this sequence of plane stress crack growth which fits into the framework of plane stress growth considered more broadly in [3]. For the sequence considered here, it is shown that the energy/area can be partitioned as $\Gamma_{0}=\Gamma_{I}+\Gamma_{I I}$ with $\Gamma_{I}$ as the energy/area dissipated between the onset of necking and the onset of shear localization and $\Gamma_{I I}$ as that dissipated in shear localization and shear fracture. The first contribution, $\Gamma_{I}$, dominates the total energy dissipated during crack advance and it scales exactly with the plate thickness, $W_{0}$, according to $\Gamma_{I} \propto \sigma_{y} W_{0}$. For a 1-centimeter thick plate made of a ductile metal with yield stress $\sigma_{y}=300 \mathrm{MPa}, \Gamma_{I} \sim 1 \mathrm{MJm}^{-2}$. By contrast, the second contribution scales as $\Gamma_{I I} \propto \sigma_{y} \ell$ where $\ell$ is the thickness of the shear localization band, which scales with the element size in the presented FE analysis; for $\sigma_{y}=300 \mathrm{MPa}$ and $\ell=30 \mu \mathrm{m}, \quad \Gamma_{I I} \sim 0.01 \mathrm{MJm}^{-2}$. This numerical example highlights the fact that, because plasticity constitutes the major portion of the dissipation for both contributions, each of them are huge compared to the atomistic work of separation, which is typically only several $\mathrm{Jm}^{-2}$. Furthermore, this example clearly demonstrates that $\Gamma_{I} \gg>\Gamma_{I I}$. 
The cohesive zone characterized in this paper is associated with a mode I crack that has propagated several plate thicknesses such that the zone ahead of the crack tip is fully developed and is advancing under nominally steady-state tearing conditions. If the crack is initially sharp when it begins to first propagate, the relevant initial toughness will be closer to the plane strain toughness than to the "plane stress" toughness that is the focus here. The work of separation for a tough ductile alloy under plane strain conditions scales according to $\Gamma_{0} \sim \sigma_{Y} D$ where $D$ is the spacing of the voids that dominate the fracture process. For plates thick enough such that the plane strain toughness (or some approximation to this toughness) governs the intitiation of crack growth, the initial fracture energy is likely to be much smaller than the plane stress fracture energy. This almost certainly implies that a cohesive zone representation expected to capture behavior initiating from an initial sharp crack will require a transition from an initial propagation phase with lower separation energy to the steady-state level with higher separation energy. It also remains for further work to determine cohesive zone parameters capable of charactering crack initiation from a stress concentration such as a notch. Further, it remains for future work to extend the characterization of a cohesive zone model for ductile plates for mixed mode in-plane tearing under conditions where the crack path will be curved. For ductile plates, the cohesive zone is likely to follow the path created by the incipient neck as it propagates ahead of the advancing crack tip. Results for the onset of sheet necking under conditions other than plane strain tension will be needed; these are available in the form of sheet metal forming limits. Finally, to be generally applicable in a large finite element code for structural analysis of plates and shells, the cohesive zone representation will have to incorporate the effects of bending moments and, possibly transverse forces, on the generalized tractionseparation behavior.

\section{Acknowledgements}

KLN is financially supported by the Danish Technical Research Council in a project entitled "Plasticity across scales". JWH is supported by an ONR MURI grant to Harvard University. 


\section{References}

[1] Roychowdhury, S., Roy, Y.D., Dodds, R.H., Ductile tearing in thin aluminum panels: experiments and analyses using large-displacement, 3-D surface cohesive elements, Eng. Fract. Mech., 2002;69:983-1002.

[2] Chabanet, O., Steglich, D., Besson, J., Heitmann, V. Hellmann, D., Brocks, W., Predicting crack growth resistance of aluminum sheets, Comp. Mater. Sci. 2003;26:1-12.

[3] Pardoen, T., Hachez. F., Marchioni, B., Blyth, P.H., Atkins, A.G., Mode I fracture of sheet metal, J. Mech. Phys. Solids 2004;53:423-452.

[4] Scheider, I., Brocks, W., Cohesive elements for thin-walled structures, Comp. Mater. Sci. 2006;37:101-109.

[5] Simonsen, B.C, Törnqvist, R., Experimental and numerical modeling of ductile crack propagation in large scale shell structures, Marine Structures, 2004;17:1-27.

[6] Gurson, A., Continuum theory of ductile rupture by void nucleation and growth - I. Yield criteria and flow rules for porous ductile media. J. Eng. Mater. Technol. 1977;9:2-15.

[7] Nahshon, K., Hutchinson, J.W., Modification of the Gurson model for shear failure, Euro. J. Mech. A/Solids, 2008;27:1-17.

[8] Tvergaard, V., Influence of voids on shear band instabilities under plane strain conditions, Int. J. Frac. 1981;17:389-407.

[9] Becker, R., Needleman, A., Effect of yield surface curvature on necking and failure in porous plastic solids, J. Applied Mech., 1986;53:491-499.

[10] Mathur, K.K., Needleman, A., Tvergaard, V., Three dimensional analysis of dynamic ductile crack growth in a thin plate, J. Mech. Phys. Solids, 1996;44:439-464.

[11] Besson, J., Steglich, D., Brocks, W., Modelling of crack growth in round bars and plane strain specimens, Int. J. Solids Struct. 2001;38:8259-8284.

[12] Besson, J., Steglich, D., Brocks, W., Modelling of plane strain ductile rupture, Int. J. Plasticity 2003;19:1517-1541.

[13] Xue, L., Wierzbicki, T., Numerical Simulation of fracture mode transition in ductile plates, Int. J. Solids Struct. 2009;46:14231435. 
[14] Tvergaard, V., On localization in ductile materials containing spherical voids, Int. J. Frac. 1982;18:287-252.

[15] Xue, Z., Pontin, M.G., Zok, F.W., Hutchinson, J.W., Calibration procedures for a computational model of ductile fracture, Eng. Frac. Mech., 2010;77:492-509.

[16] Tvergaard, V. and Nielsen, K.L., Relations between a micro-mechanical model and a damage model for ductile failure in shear, J. Mech. Phys. Solids, 2010, doi:10.1016/j.jmps.2010.06.006.

[17] Budiansky, B., Remarks on Theories of solid and structural mechanics. Harvard University, SIAM, p. 77-83.

[18] Hutchinson, J., Finite strain analysis of elastic-plastic solids and structures. Harvard University, AMD, 1973;6:17-29.

[19] Tvergaard, V., Necking in tensile bars with rectangular cross-section, Comp. Methods in Appl. Mech. Eng. 1993;103:273-290.

[20] Zienkiewicz, O.C. and Taylor, R.L., The Finite Element Method - The Basis, Vol. 1, 5. Edition, Butterworth-Heinemann, 2000.

[21] Rice, J.R., 1977. The localization of plastic deformation. In: Koiter, W.T. (Ed.), Theoretical and Applied Mechanics, vol. 1. North-Holland Publishing, Delft, pp. 207-220.

[22] Tvergaard, V., Hutchinson, J.W., The relation between crack growth resistance and fracture process parameters in elastic-plastic solids, J. Mech. Phys. Solids, 1992;40:1377-1397. 


\section{Figures}

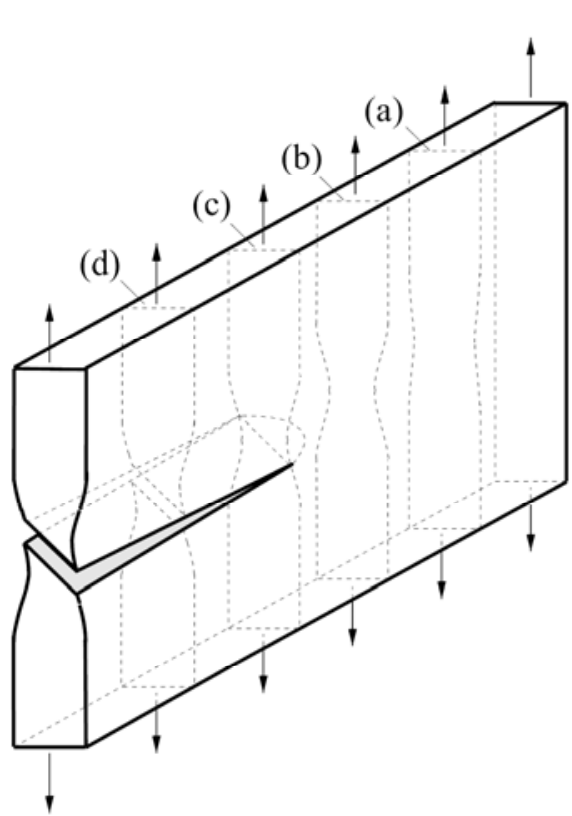

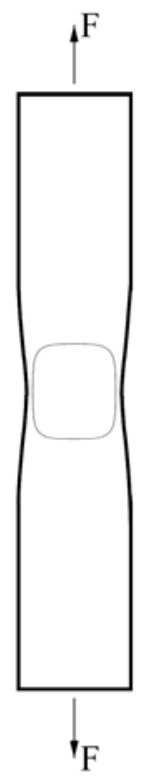

(a)

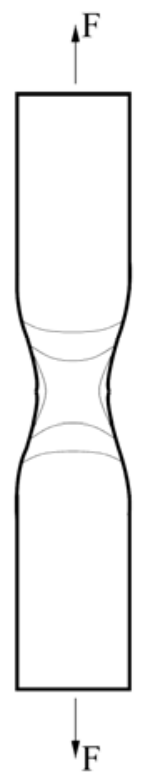

(b)

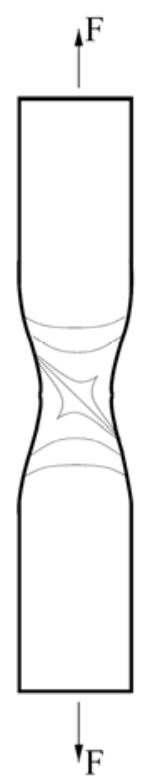

(c)

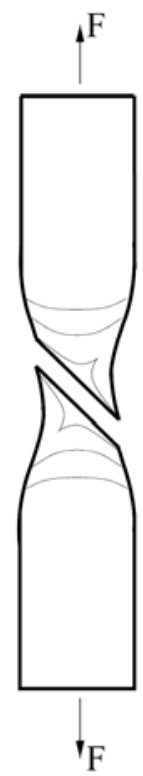

(d)

Figure 1: Sequential fracture process governing crack advance in ductile sheet metal subject to mode I loading, (a) onset of local necking, (b) local thinning, (c) shear localization and (d) slant failure.

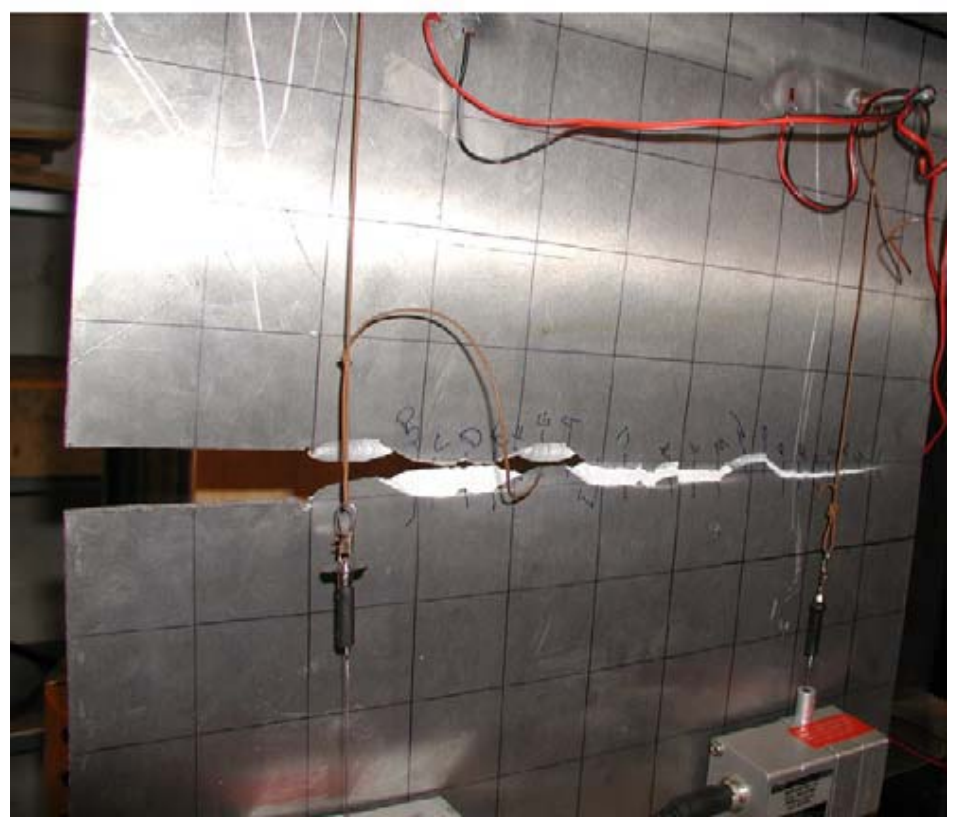

Figure 2: A 10mm thick plate of A5083 aluminum tested by Simosen and Törnqvist [5]. The crack was initiated at the edge notch located on the left edge of the plate. Flipping of the slant fracture from one 45 degree orientation to the other is evident. 


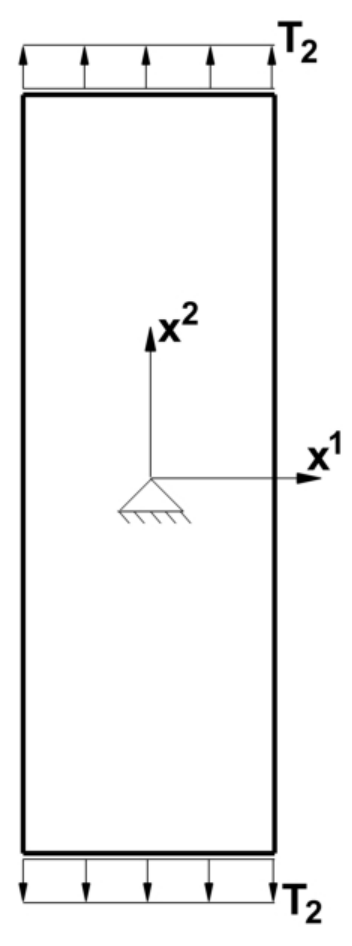

(a)

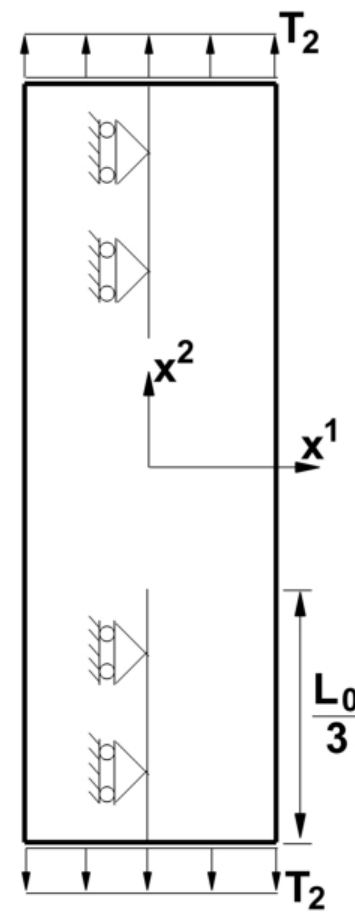

(b)

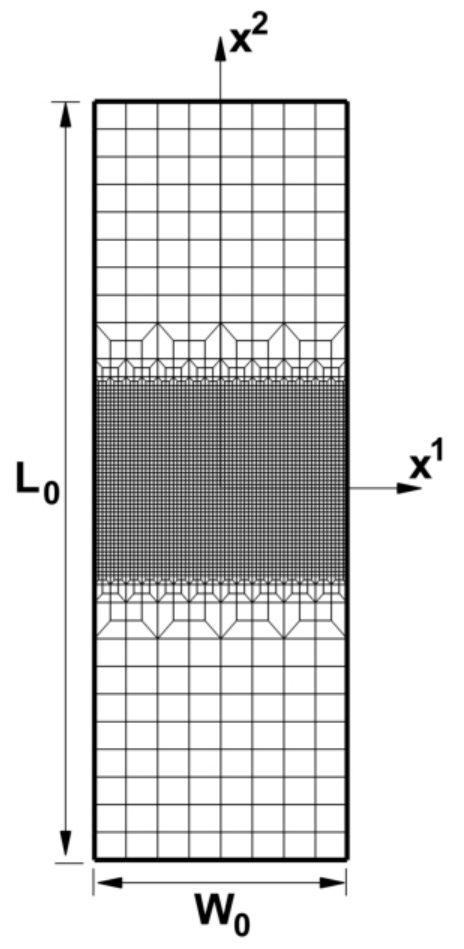

(c)

Figure 3: Schematic illustrating boundary conditions, notation and mesh, (a) unconstrained condition, (b) constrained condition, and (c) representative mesh (element size $L^{(e)}=W_{0} / 64$ ). 


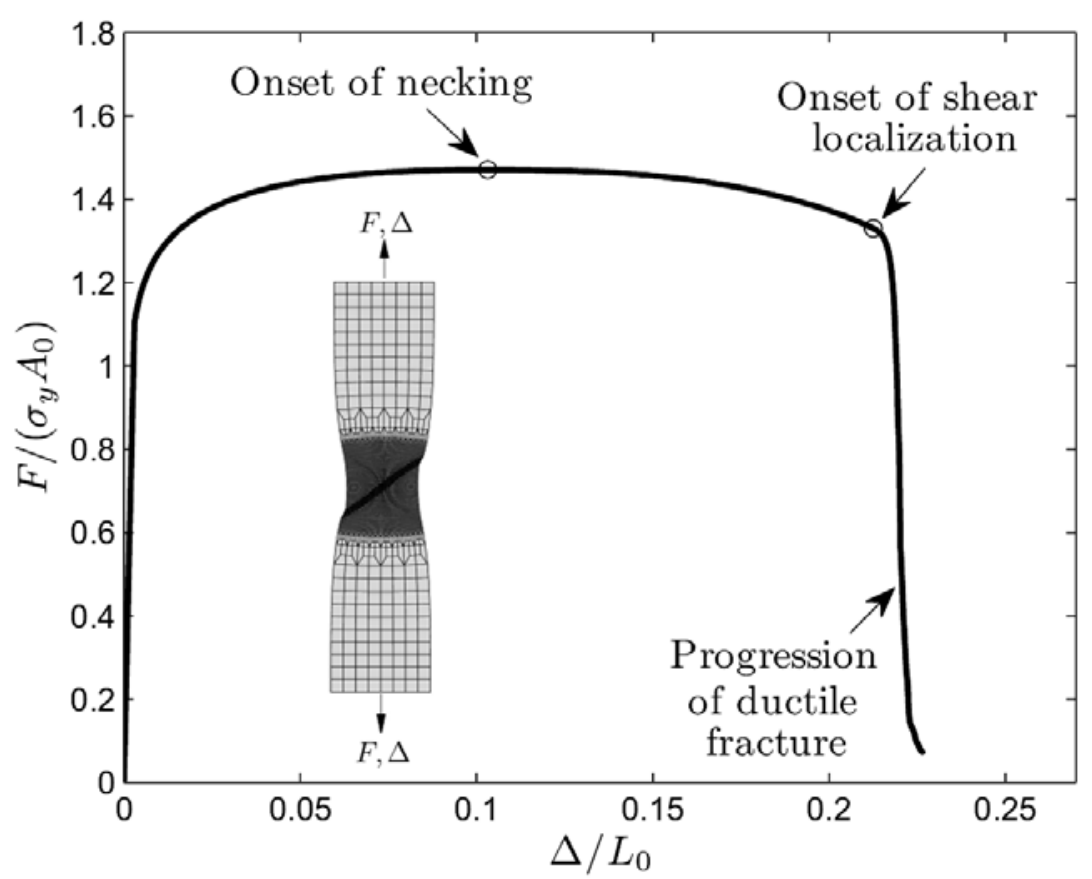

(a)

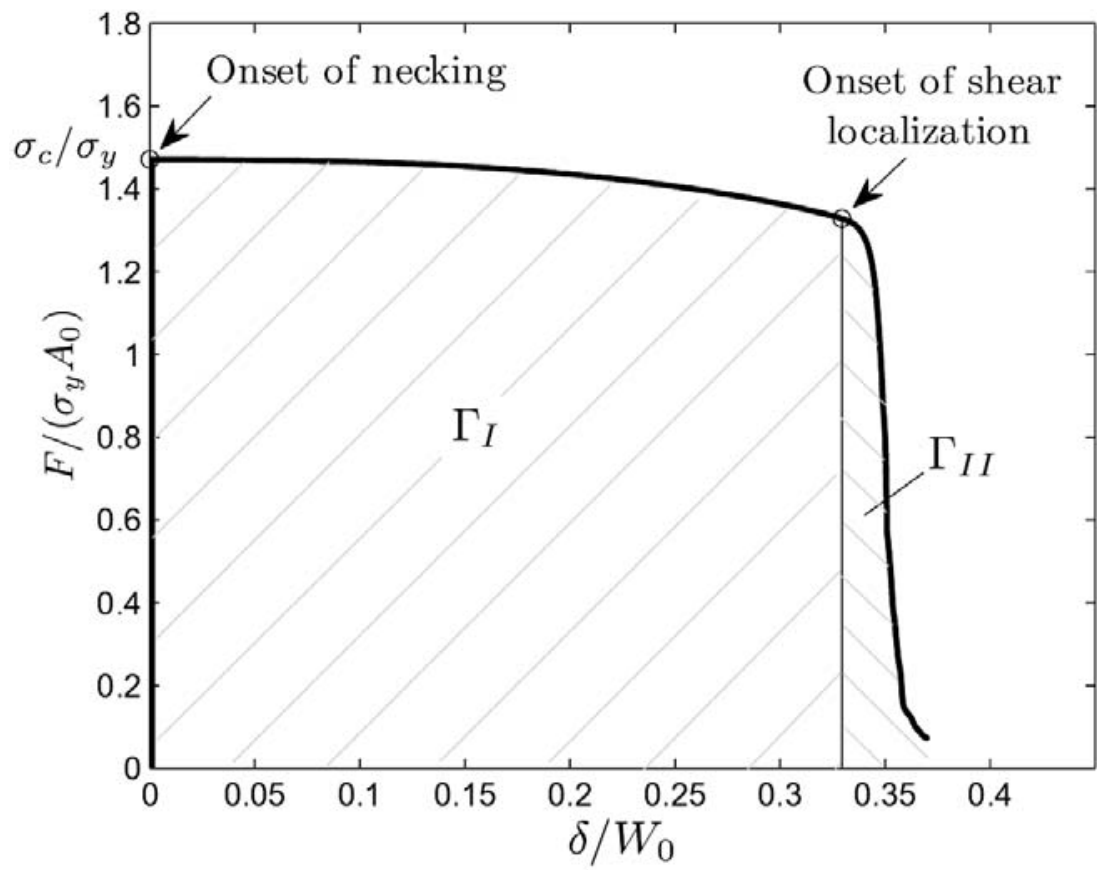

(b)

Figure 4: (a) Normalized overall load-deflection curve for the metal sheet section considered, and (b) traction-separation curve for the cohesive zone extracted from Fig. (a), with indicated fracture energy associated with necking, $\Gamma_{I}$, and shear localization and fracture, $\Gamma_{I I}$, respectively. 


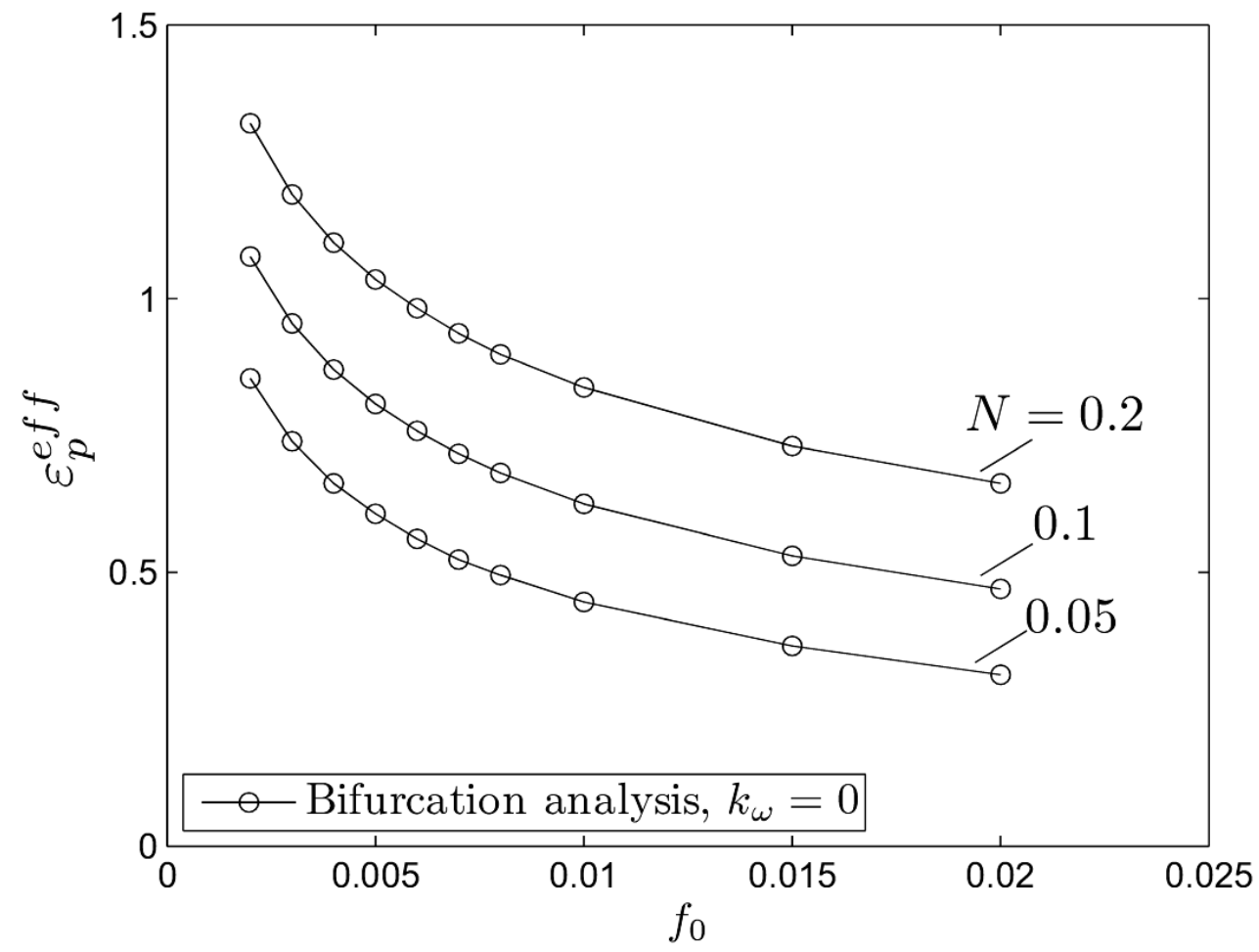

(a)

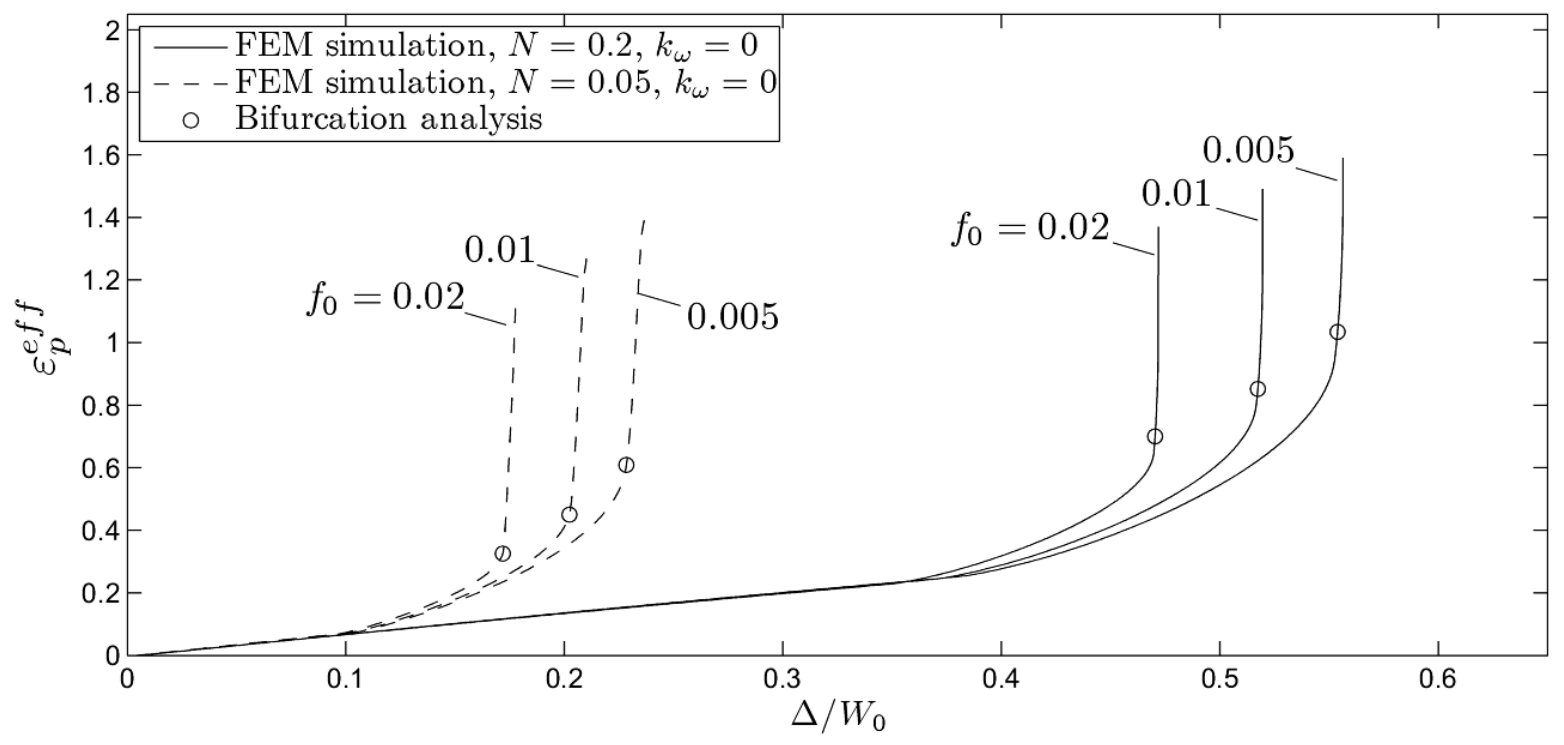

(b) 


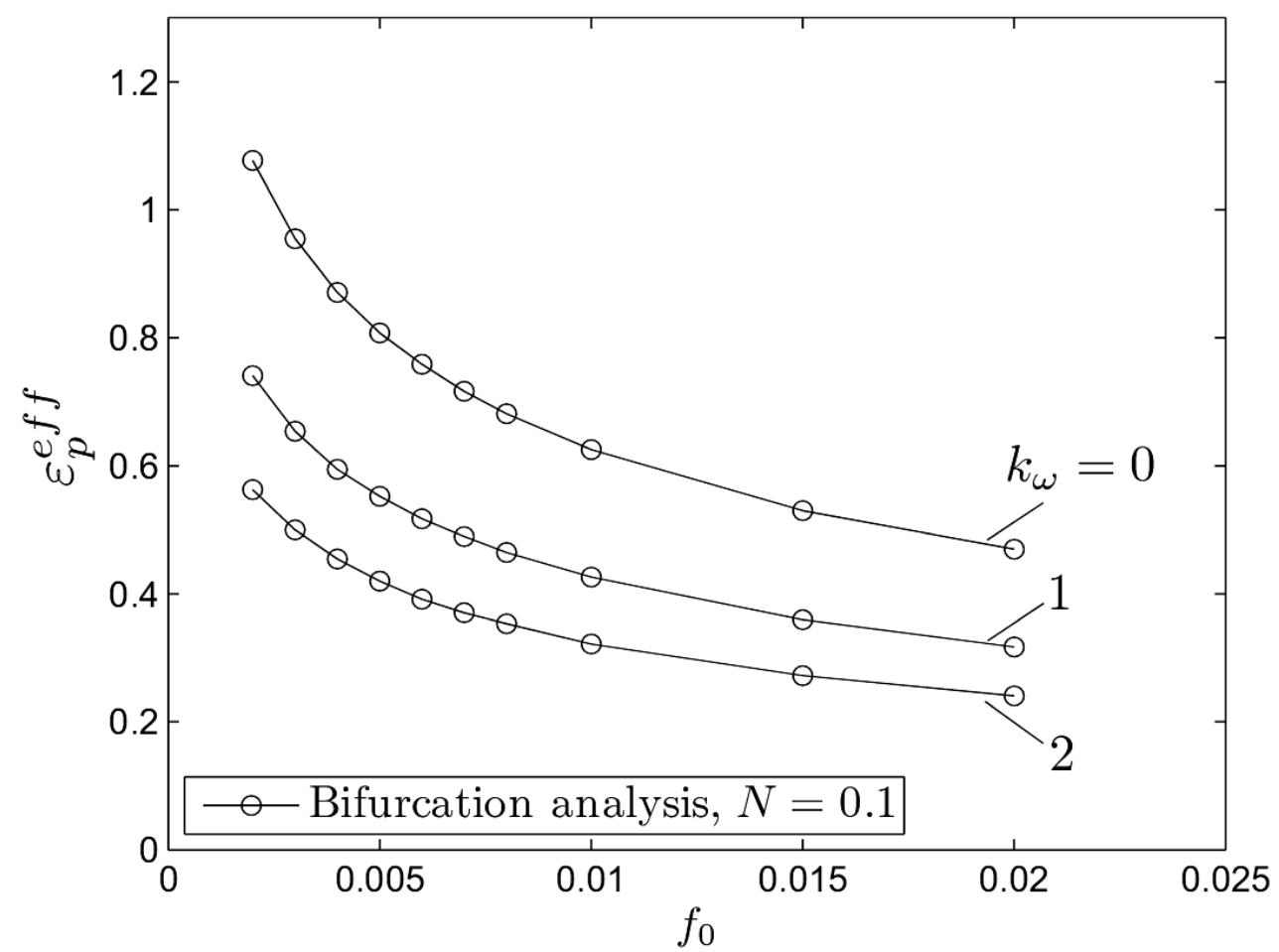

(c)

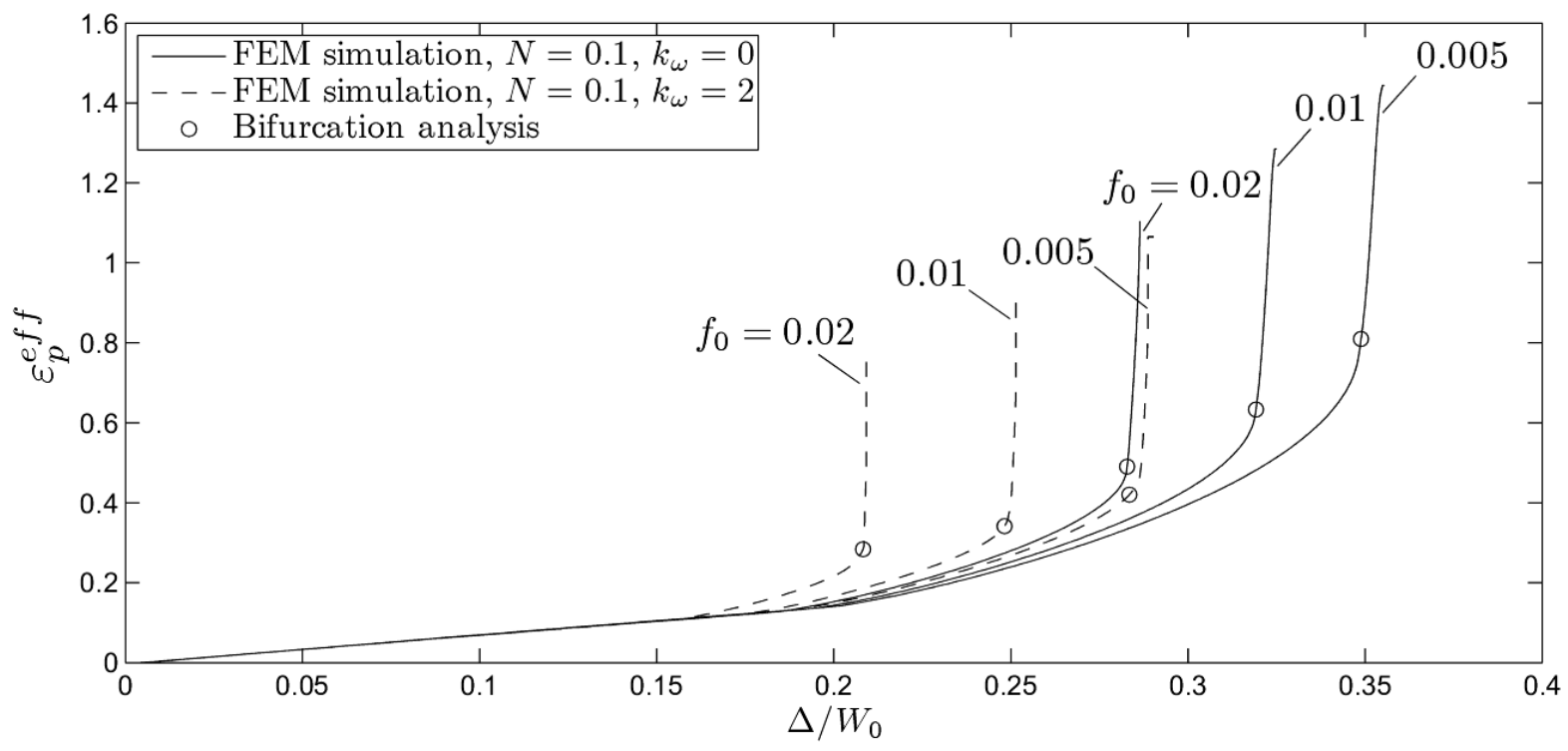

(d)

Figure 5: Effective plastic strain at shear localization based on $(a, c)$ bifurcation analysis in plane strain tension, and comparison with the local effective plastic strain predicted using the FE-model at $\left(x_{1}, x_{2}\right)=(0,0)$, for (b) $k_{\omega}=0$ for $N \in[0.05,0.2]$ and $f_{0} \in[0.005,0.02]$ and (d) $k_{\omega} \in[0,2]$ for $N=0.1$ and $f_{0} \in[0.005,0.02] .\left(\sigma_{y} / E=0.003\right)$ 


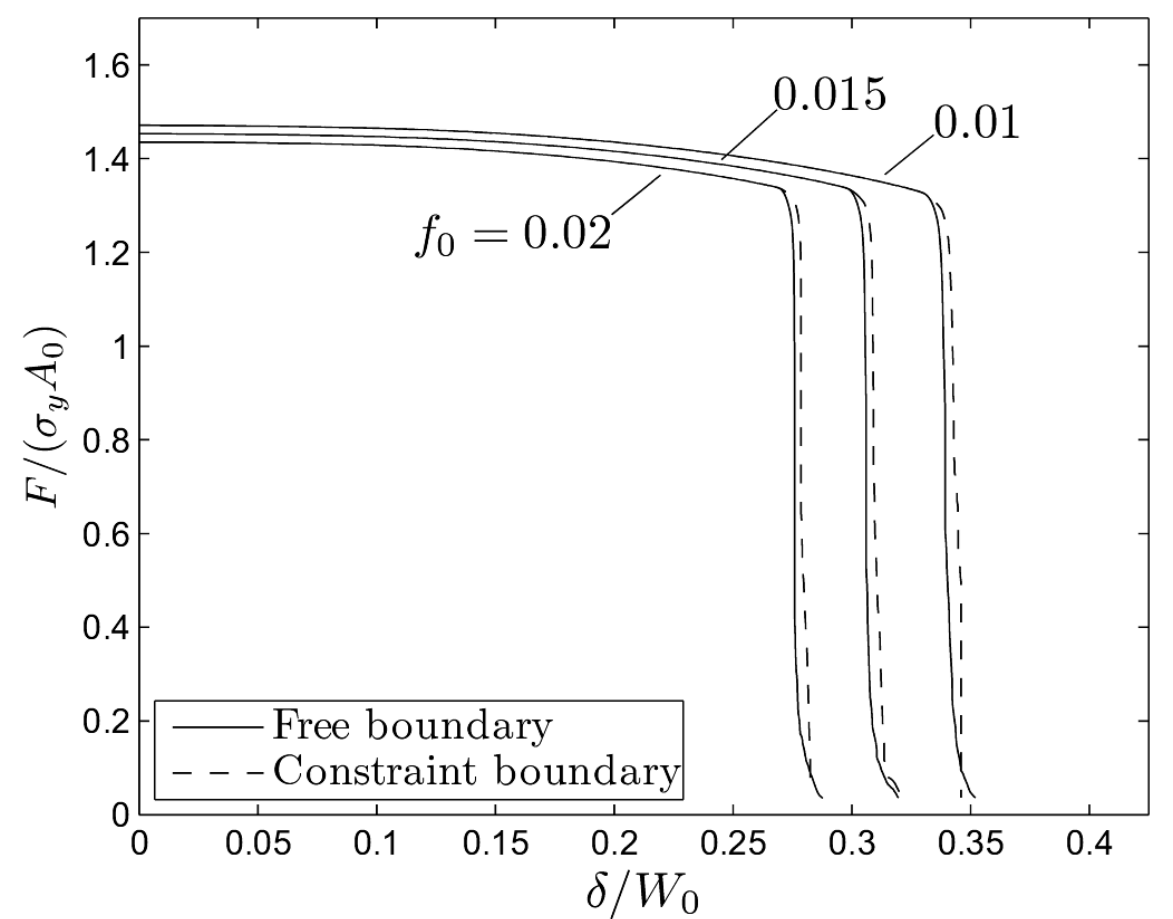

(a)

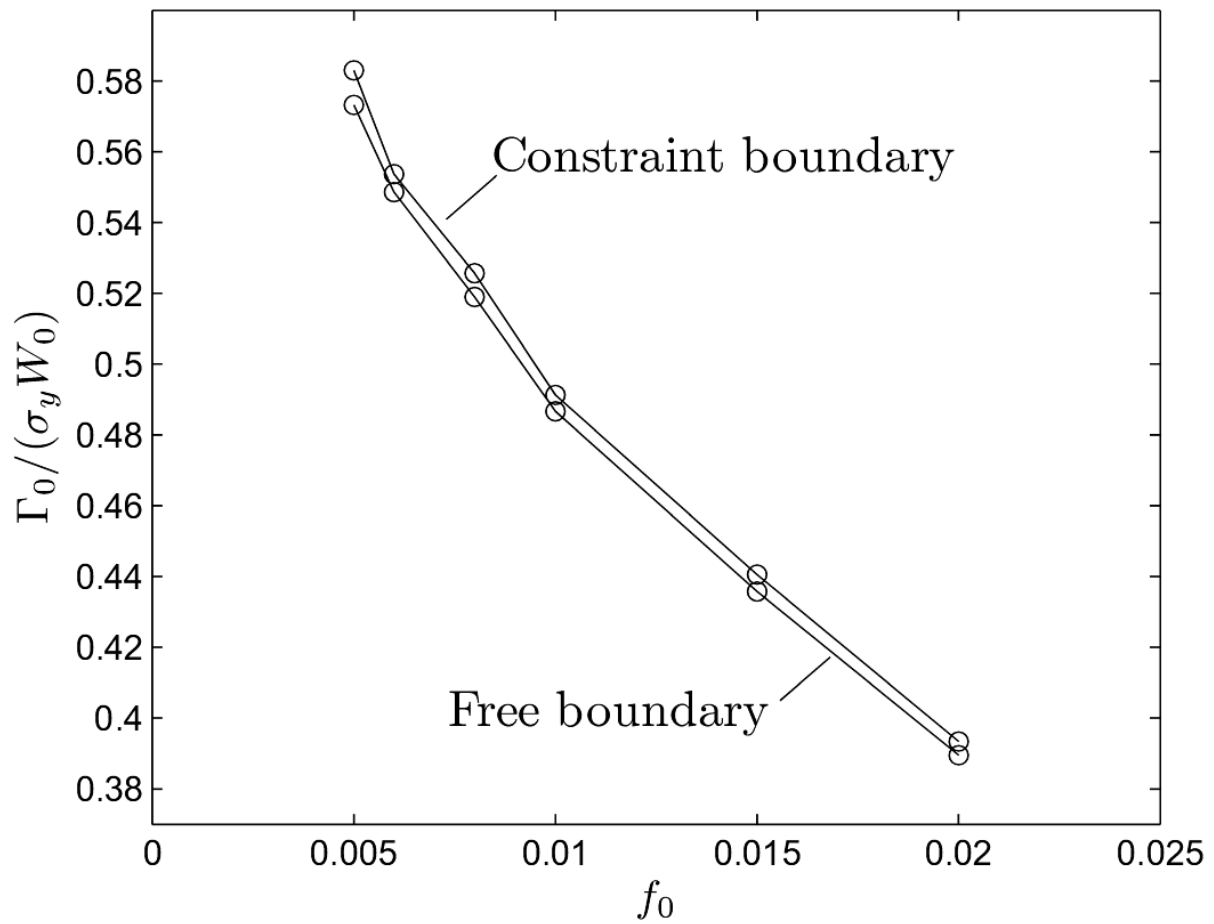

(b)

Figure 6: Effect of the out-of-plane deflection constraint across the fracture zone: (a) normalized overall load-deflection curves for constrained and unconstrained sections and (b) associated cohesive energy, $\Gamma_{0}=\Gamma_{I}+\Gamma_{I I}$, for $f_{0} \in[0.005,0.02], N=0.1, k_{\omega}=0$ and $\sigma_{y} / E=0.003$. 


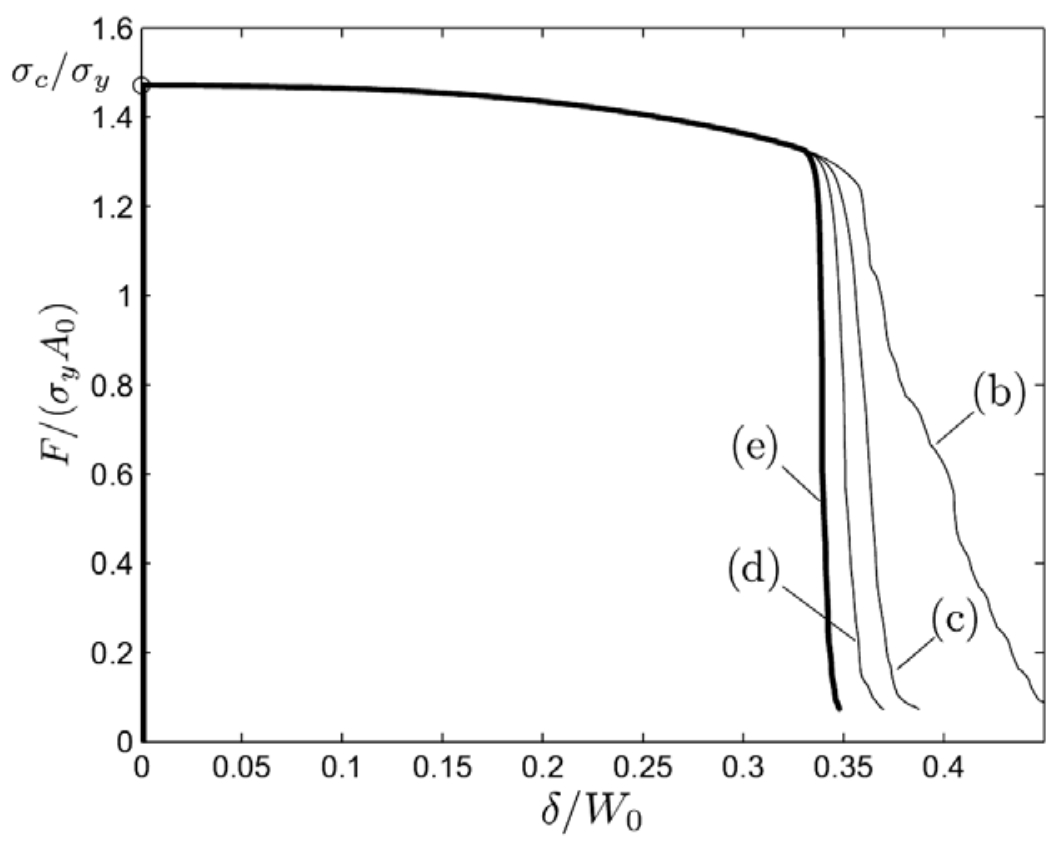

(a)

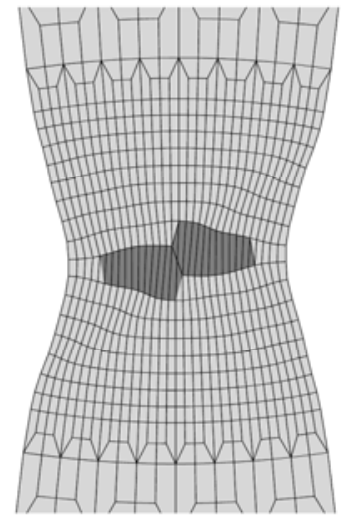

(b)

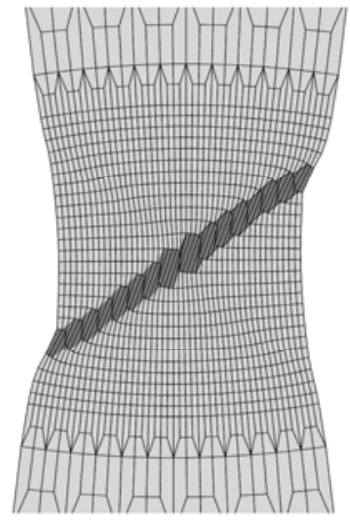

(c)



(d)

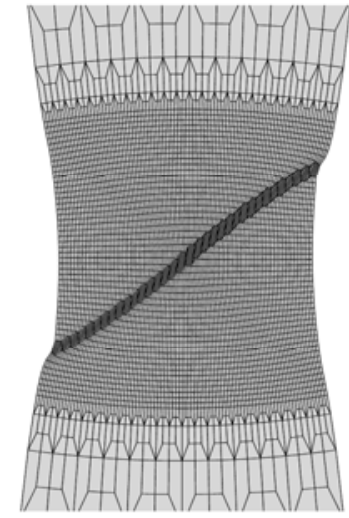

(e)

Figure 7: Mesh dependence: (a) element size effect on traction-separation curve, and associated failure modes for element of size (b) $L^{(e)}=W_{0} / 32$, (c) $L^{(e)}=W_{0} / 48$, (d) $L^{(e)}=W_{0} / 64$, and (e) $L^{(e)}=W_{0} / 96\left(\sigma_{y}=630 \mathrm{MPa}, N=0.1, k_{\omega}=0, f_{0}=0.01\right)$ 


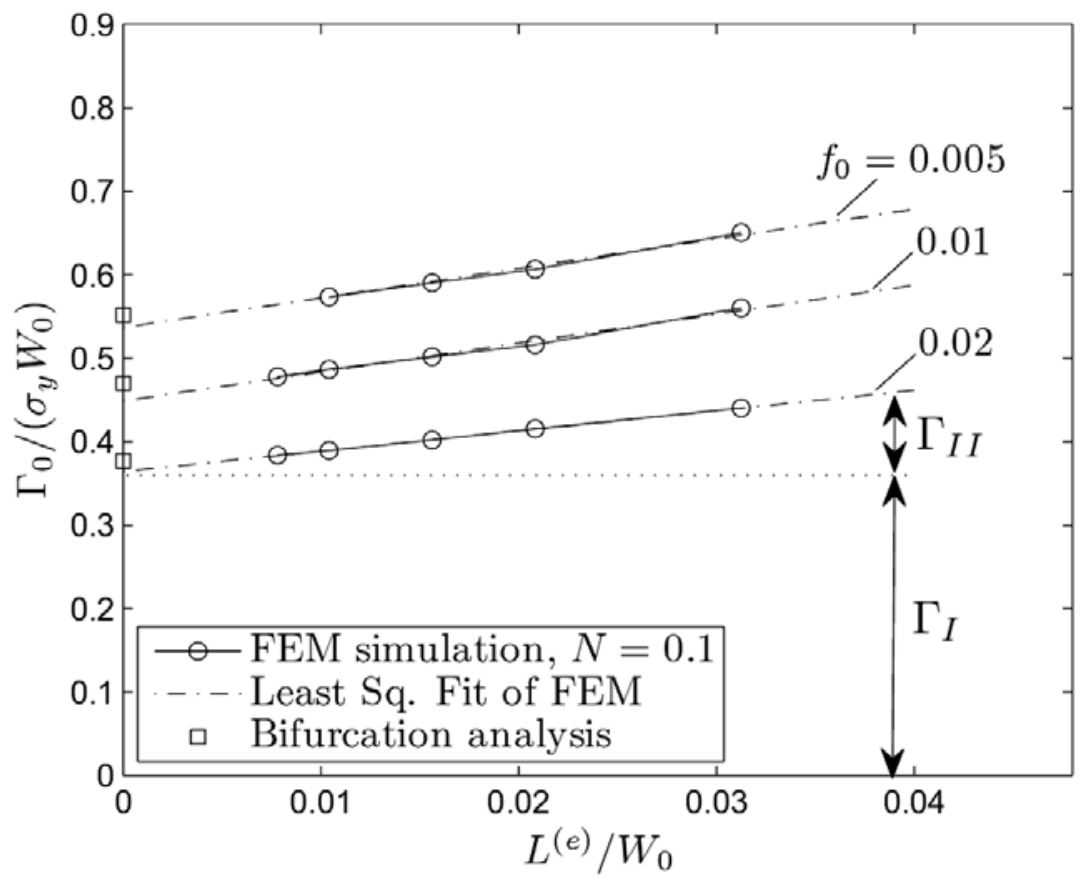

(a)

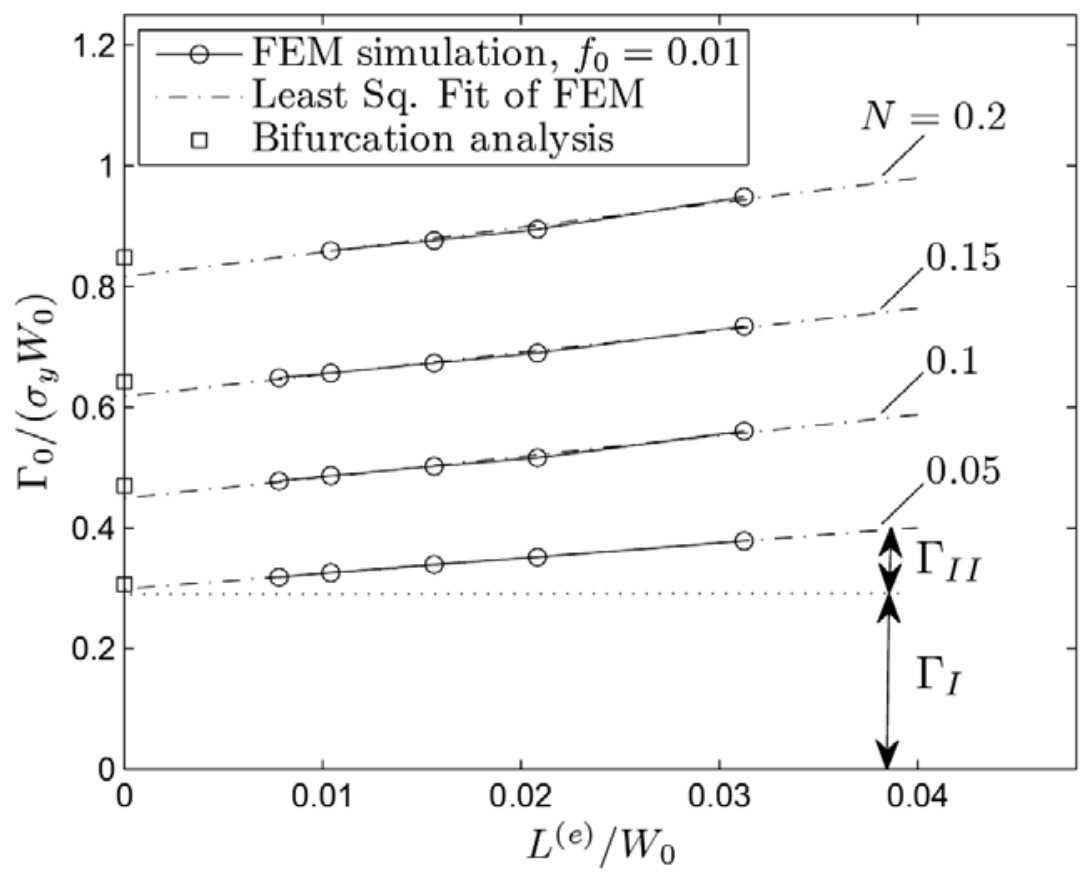

(b)

Figure 8: Cohesive energy dependency on element size during sheet necking, shear localization and fracture, showing the effect of (a) Initial porosity $f_{0} \in[0.005,0.02]$ with $N=0.1$, and (b) Strain hardening $N \in[0.05,0.2]$ with $f_{0}=0.01$. The other parameters are the same as those specified in Figure 7. 


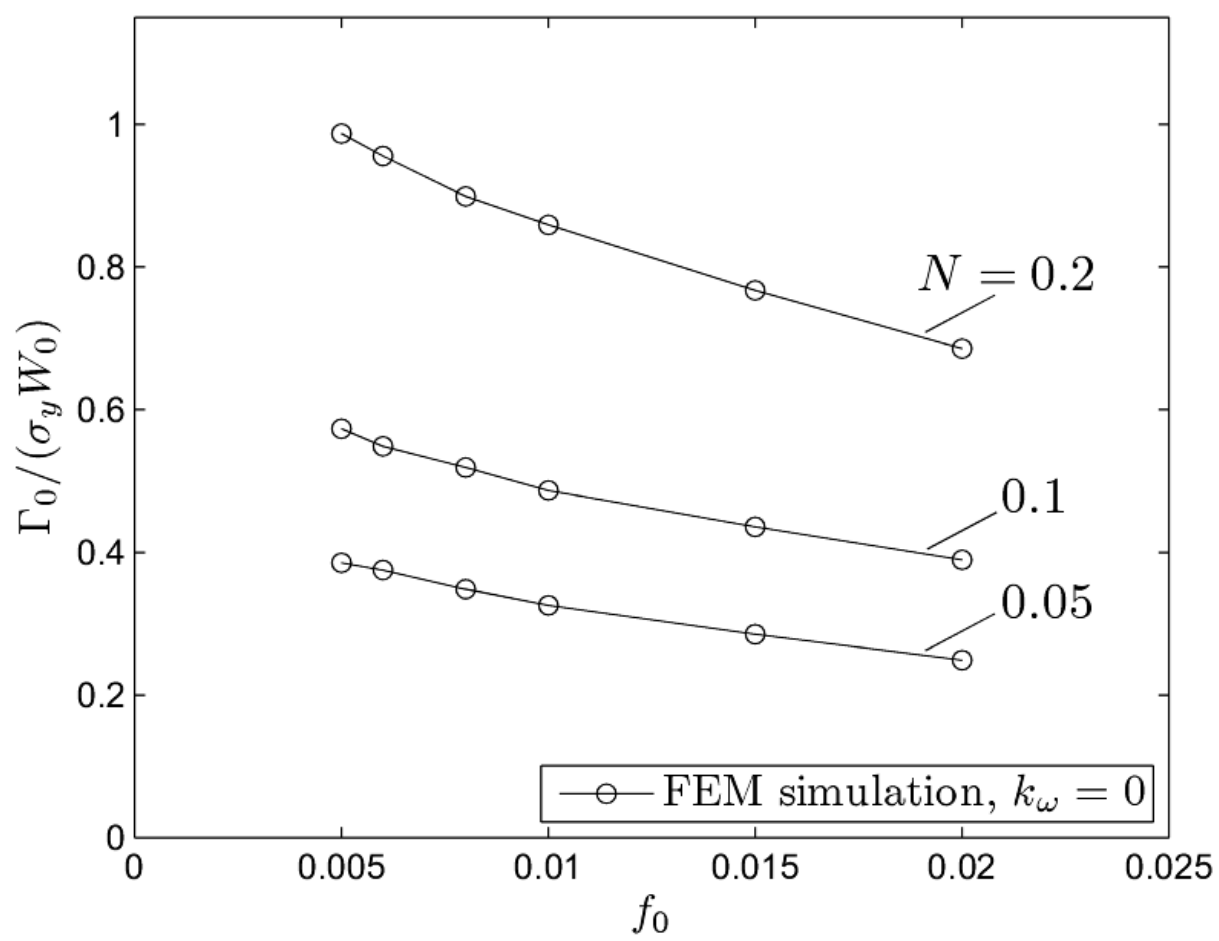

(a)

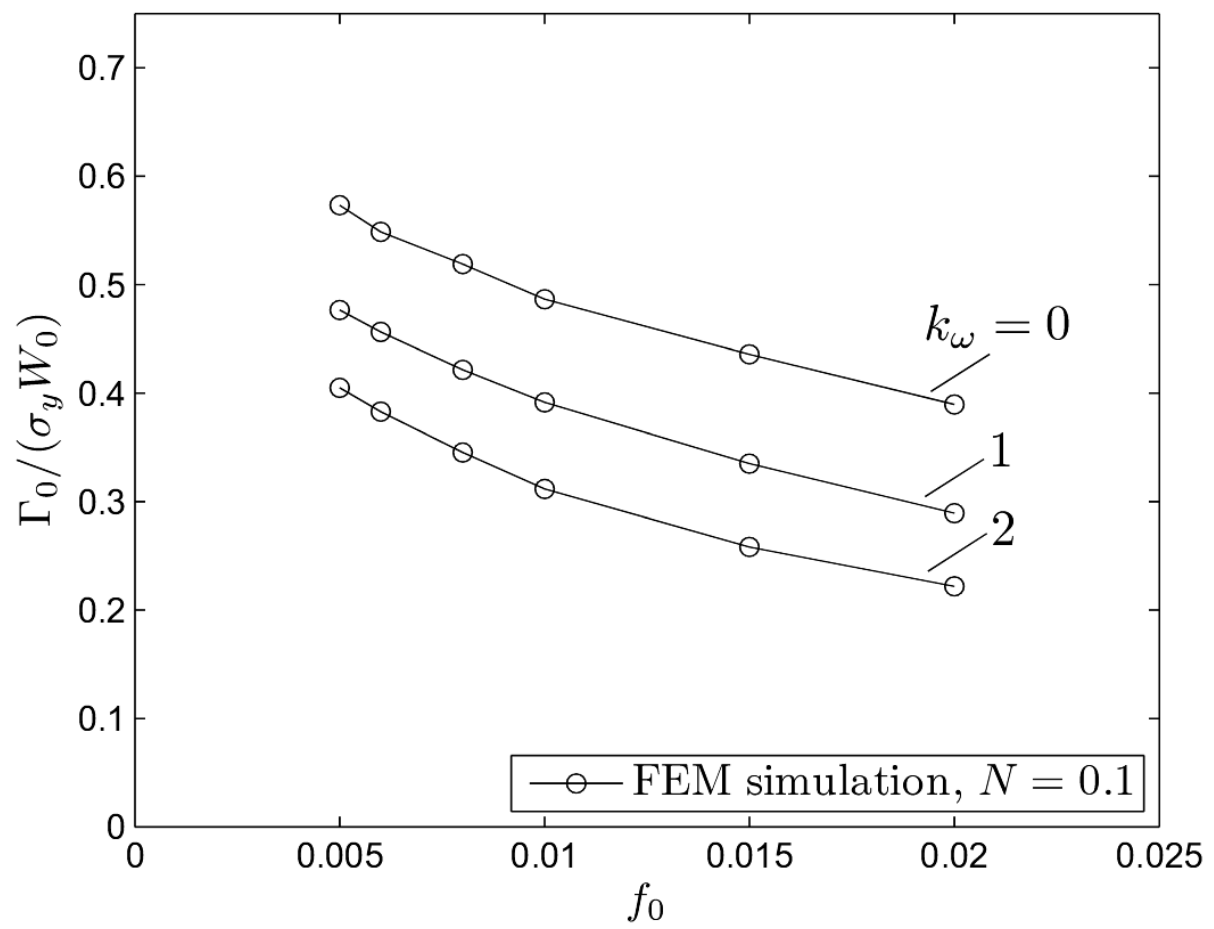

(b)

Figure 9: Cohesive energy as a function of initial void volume fraction showing the effect of (a) strain hardening $N \in[0.05,0.2]$ with $k_{\omega}=0$ and (b) the shear damage coefficient $k_{\omega} \in[0,2]$ with $N=0.1$. The other parameters are the same as those specified in Figure 7. 


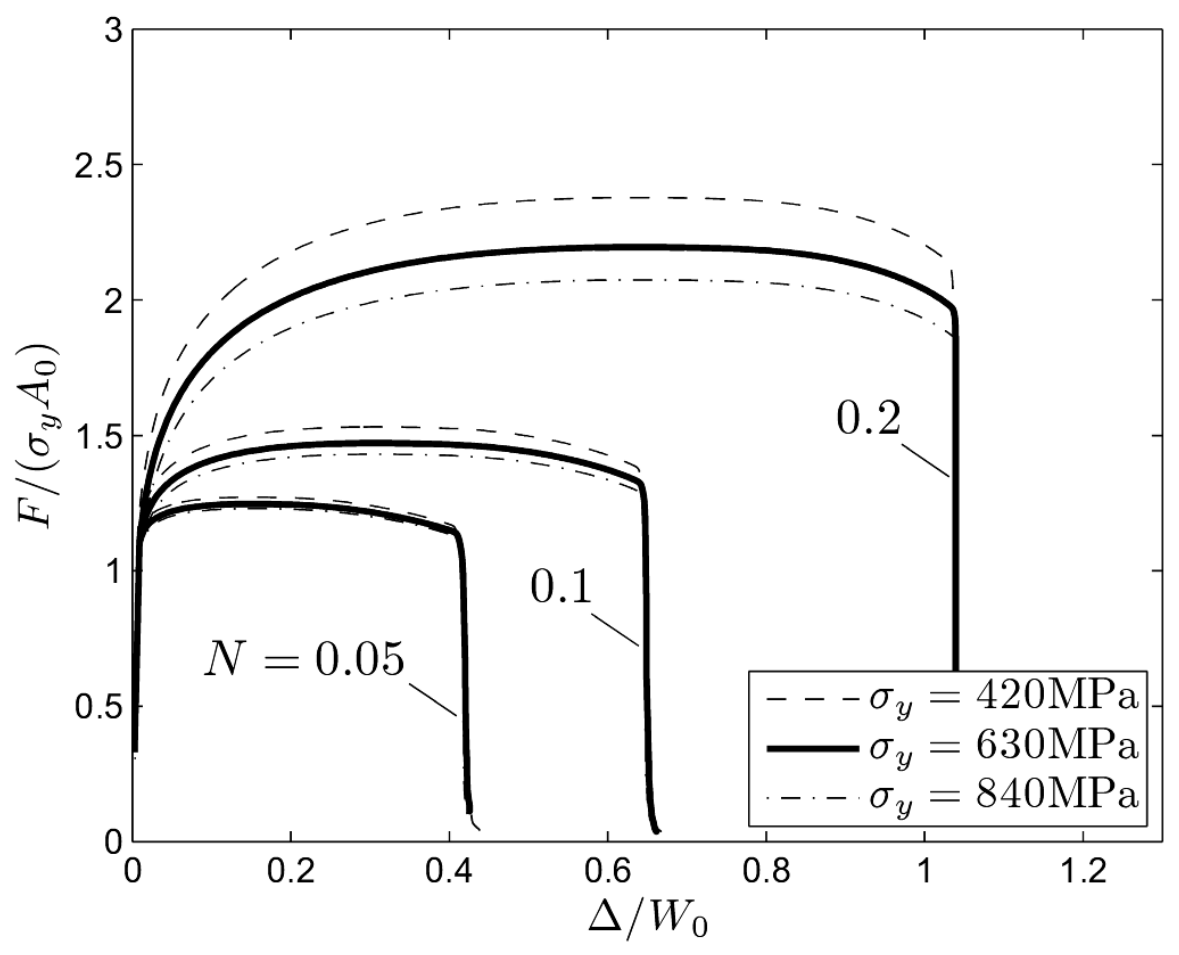

(a)

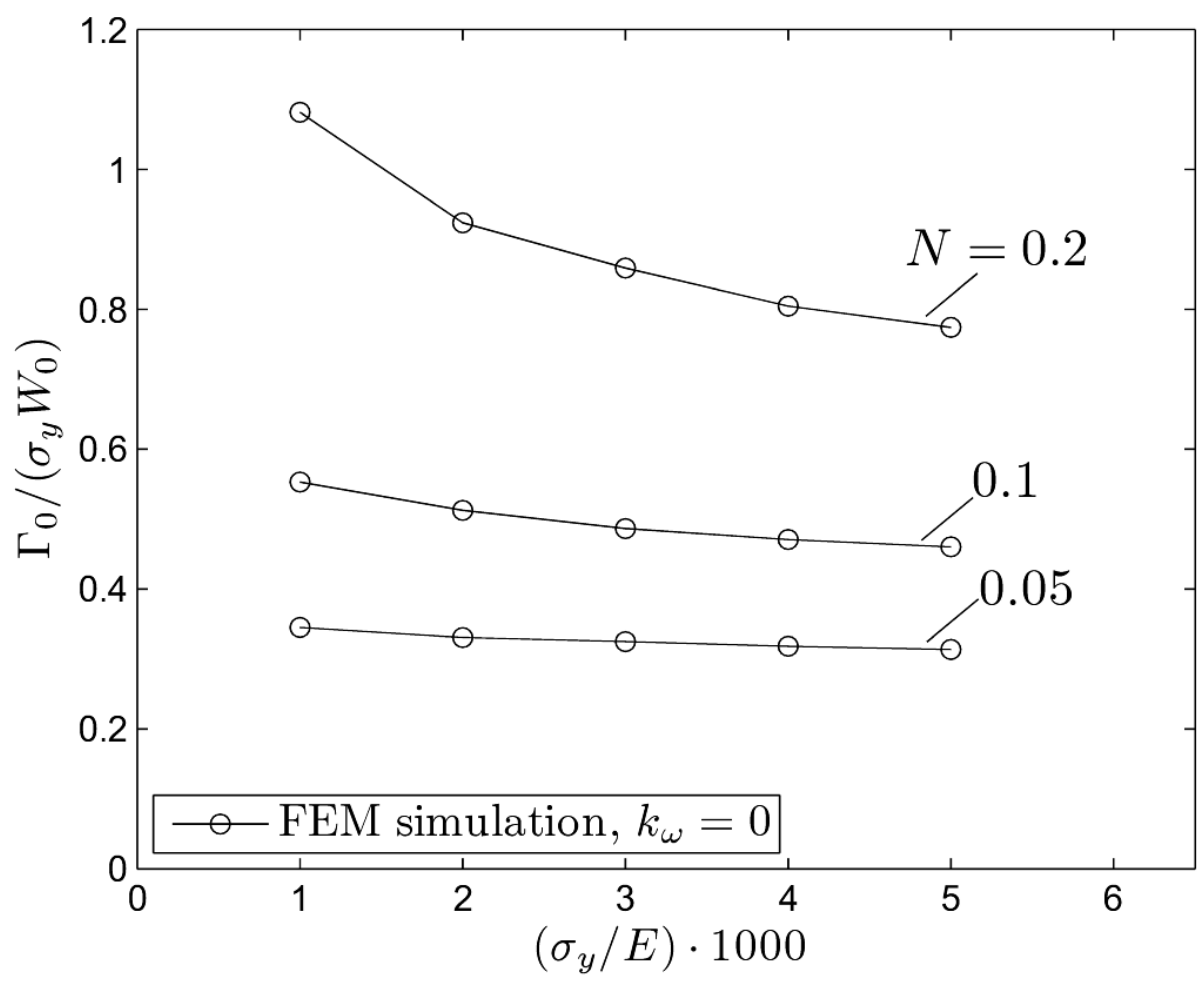

(b) 


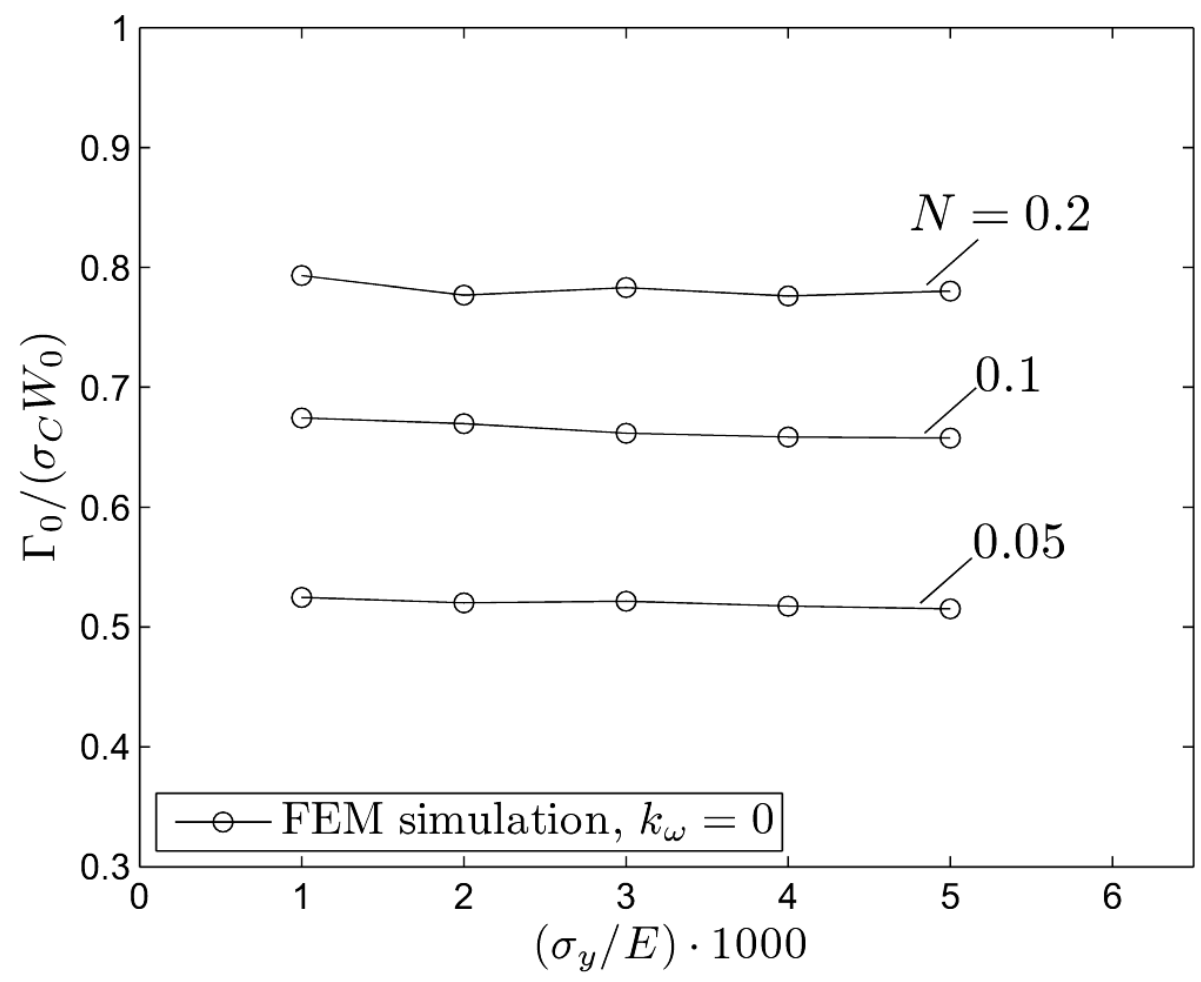

(c)

Figure 10: (a) Normalized overall load-deflection curves for metal sheet section showing the effect of $\sigma_{y} / E$. (b) Cohesive energy normalized using the yield stress, and (c) Cohesive energy normalized using the nominal Considère stress, $\sigma_{C}\left(N \in[0.05,0.2], k_{\omega}=0, f_{0}=0.01\right)$. 


\section{Tables}

\begin{tabular}{lll}
\hline Parameters & Notation & Value \\
\hline Youngs modulus & $E$ & $210 \mathrm{GPa}$ \\
Poisson ratio & $v$ & 0.3 \\
Yield stress & $\sigma_{y}$ & $630(210-1050) \mathrm{MPa}$ \\
Strain hardening & $N$ & $0.05-0.2$ \\
Initial porosity & $f_{0}$ & $0.005-0.02$ \\
Yield surface constants & $q_{1}, q_{2}$ & $1.5,1$ \\
Critical void volume fraction & $f_{c}$ & 0.15 \\
Final void volume fraction & $f_{f}$ & 0.40 \\
Shear coefficient & $k_{\omega}$ & $0-2$ \\
\hline
\end{tabular}

Table 1: Material properties and damage parameters. 